\title{
Network Pharmacology Analysis of ZiShenWan for Diabetic Nephropathy and Experimental Verification of Its Anti-Inflammatory Mechanism
}

This article was published in the following Dove Press journal:

Drug Design, Development and Therapy

\author{
Xiaoyuan Guo $\mathbb{D}^{\prime}$ \\ You Wu (iD) ${ }^{2}$ \\ Chengfei Zhang ${ }^{2}$ \\ Lili $\mathrm{Wu}^{3}$ \\ Lingling Qin $^{4}$ \\ Tonghua Liu (iD) ${ }^{3}$ \\ 'Department of Nephrology, Dong Fang \\ Hospital, Beijing University of Chinese \\ Medicine, Beijing, 100078, People's \\ Republic of China; ${ }^{2}$ Second Clinical \\ Medical College, Beijing University of \\ Chinese Medicine, Beijing, I00078, \\ People's Republic of China; ${ }^{3}$ Key \\ Laboratory of Health Cultivation of the \\ Ministry of Education, Beijing University \\ of Chinese Medicine, Beijing, 100029, \\ People's Republic of China; ${ }^{4}$ Technology \\ Department, Beijing University of \\ Chinese Medicine, Beijing, I00029, \\ People's Republic of China
}

Background: Diabetic nephropathy (DN) is the leading cause of end-stage renal disease (ESRD). The inflammatory response plays a critical role in DN. ZiShenWan (ZSW) is a classical Chinese medicinal formula with remarkable clinical therapeutic effects on DN, but its pharmacological action mechanisms remain unclear.

Aim: In this study, a network pharmacology approach was applied to investigate the pharmacological mechanisms of ZSW in DN therapy. Based on the results of network analysis, the core targets and signaling pathways related to anti-inflammatory effect were verified via experiments in vivo.

Methods: The candidate chemical ingredients of ZSW as well as its putative targets and known therapeutic targets of DN were acquired from appropriate databases. The "herbingredient-target" network for ZSW in DN treatment was established. The protein-protein interaction (PPI) network of potential targets was constructed to screen the core targets. Gene ontology (GO) and Kyoto Encyclopedia of Genes and Genomes (KEGG) pathway enrichment analyses were performed. In addition to biochemical and pathological indicators, the core targets and signaling pathways associated with inflammation were partially validated in $\mathrm{db} / \mathrm{db}$ mice at molecular level.

Results: A total of 56 active ingredients in ZSW and 166 DN-related targets were selected from databases. A high proportion of core targets and top signaling pathways participate in inflammation. ZSW markedly alleviated renal injuries pathologically and regulated related biomarkers. In particular, ZSW significantly inhibited the exaggerated release of inflammatory cytokines such as interleukin (IL)-1 $\beta$, IL-6, tumor necrosis factor receptor (TNF)-a, and monocyte chemotactic protein (MCP)-1 as well as regulating p38 mitogen-activated protein kinases (MAPK) and phosphoinositide 3-kinase (PI3K)-protein kinase B (Akt) signaling pathways in $\mathrm{db} / \mathrm{db}$ mice.

Conclusion: This study first comprehensively investigated the active ingredients, potential targets, and molecular mechanism of ZSW as a therapy for DN. ZSW achieved renoprotective effects in DN via regulation of multiple targets and signaling pathways, especially by alleviating inflammation. Results indicate that $\mathrm{ZSW}$ is a promising multi-target therapeutic approach for DN treatment.

Keywords: ZiShenWan, network pharmacology, diabetic nephropathy, inflammation

\section{Introduction}

Diabetic nephropathy (DN), the most common complication in diabetes mellitus patients, is the leading cause of end-stage renal disease (ESRD). ${ }^{1}$ DN increases mortality and imposes a great physical and mental burden as well as an economic
Key Laboratory of Health Cultivation of the Ministry of Education, Beijing University of Chinese Medicine, II Bei San Huan Dong Lu, Chaoyang, Beijing, 100029, People's Republic of China

Tel +8601064286426

Fax+86010642138I7

Email thliu@vip.163.com
Drug Design, Development and Therapy 2021:15 1577-1594

bmit your manuscrip

DovePres: $f$ in - 
burden to individual families and society. ${ }^{2} \mathrm{DN}$ is clinically characterized by varying degrees of proteinuria, which can be partially alleviated by current therapies in the early stage. However, progression accelerates after marked proteinuria appears. ${ }^{3,4}$ Therefore, the need to find effective strategies for the prevention and treatment of DN remains urgent.

Inflammation response is active throughout DN progression and plays a pivotal role especially in the early stage-participating in multiple pathological processes, including podocyte injury, glomerular basement membrane (GBM) thickening, mesangial cell proliferation, extracellular matrix (ECM) accumulation and epithelial-mesenchymal transition (EMT) in renal tubule epithelial cells. It is stimulated by inflammatory cytokines and involves genes related to monocyte chemoattractant protein (MCP)- 1 , tumor necrosis factor (TNF), interleukin (IL) 6, IL-18, and so on, as well as signaling pathways such as Janus kinase-signal transducers and activators of transcription (JAK-STAT) pathway, the nuclear transcription factor kappa B (NF- $\mathrm{B})$, and the nuclear factor erythroid 2-related factor 2 (Nrf2). ${ }^{5}$ Inflammation has recently become a popular research focus in the mechanism of DN and has provided emerging targets for the development of DN therapy.

Traditional Chinese medicine (TCM) is a comprehensive medicinal system, and its application has gradually increased worldwide, with its superior therapeutic efficacy and minimal side effects. ZiShenWan (ZSW), alternatively called TongGuanWan, which consists of Anemarrhenae Rhizoma (Anemarrhena asphodeloides Bunge., Zhimu), Phellodendri Chinensis Cortex (Phellodendron chinense Schneid., Huangbai) and Cinnamomum Cassia (Cinnamomum cassia Presl, Rougui), is a classical Chinese medicinal prescription. It was first recorded in a famous ancient medicine treatiseLanShiMiCang, written by $\mathrm{Li}$ Gao during the Yuan Dynasty. ${ }^{6}$ It was described as a prescription for treating diseases with the syndrome of "Yin-deficiency and Heatexcess". " As wasting and thirsting named "XiaoKe" in TCM is the typical syndrome of "Yin-deficiency with Heat-excess" and similar to diabetes mellitus, these three major components of ZSW were widely used in clinical practice, ${ }^{8}$ and their respective advantages including lowing blood glucose and renoprotection were investigated via experimental verifications. ${ }^{9-11}$ Notably, a previous study found that ZSW lowered the blood glucose and glycosylated hemoglobin levels as well as improved the glucose tolerance in $\mathrm{db} / \mathrm{db}$ mice, which indicated that ZSW as a TCM compound formula exhibited antidiabetic activity. ${ }^{12}$ Besides, it was shown that ZSW inhibited IL and regulated NF-KB pathway, suggesting an anti-inflammatory activity. ${ }^{7}$ The main substances of ZSW compound including mangiferin, berberine, cinnamic aldehyde, timosaponin BII, and timosaponin AIII have been identified and quantified, ${ }^{12}$ some of which exerted an anti-inflammatory effect via regulating signaling pathways mentioned above. ${ }^{13-15}$ However, because the ingredients of TCMs are diverse and the interactions of TCMs with the human body are complex, the specific molecular mechanisms by which ZSW acts on DN as a TCM compound are unclear. Network pharmacology, as a breakthrough approach in the application of bioinformatics and systems biology to TCM, has sparked new interest and become a powerful strategy for research on TCM formulas. ${ }^{16}$

This study aims to investigate the therapeutic effects of ZSW on DN and examine the underlying mechanisms via a network pharmacology-based approach. Considering the importance of inflammation in DN, the anti-inflammatory mechanism was verified in vivo using $\mathrm{db} / \mathrm{db}$ mice. The flowchart of the study is shown in Figure 1.

\section{Materials and Methods}

\section{Network Pharmacology Analysis}

Database Building and Prediction of Potential Targets

The candidate chemical ingredients of the three herbs contained in ZSW and the putative targets of these ingredients were identified with the Traditional Chinese Medicine Systems Pharmacology (TCMSP, http://1sp.nwu.edu.cn/tcmsp.php, updated on May 31, 2014) database using "Anemarrhenae Rhizoma", "Phellodendri Chinensis Cortex" and "Cinnamomum Cassia" as the search terms. ${ }^{17}$ The candidate active ingredients were screened by absorption, distribution, metabolism, and excretion (ADME) properties such as oral bioavailability (OB) and drug similarity (DL). ${ }^{16}$ $\mathrm{OB} \geq 30 \%$ and $\mathrm{DL} \geq 0.18$ were used as the filtering criteria. Besides, although with low OB and DL, cinnamic acid and cinnamyl acetate were also listed as candidate compounds considering their abundance in Cinnamomum cassia. ${ }^{15}$

The known therapeutic targets acting on DN were searched and collected from databases including the Comparative Toxicogenomic Database (CTD, http:// ctmase.org/, updated on June 2, 2020), ${ }^{18}$ Therapeutic 


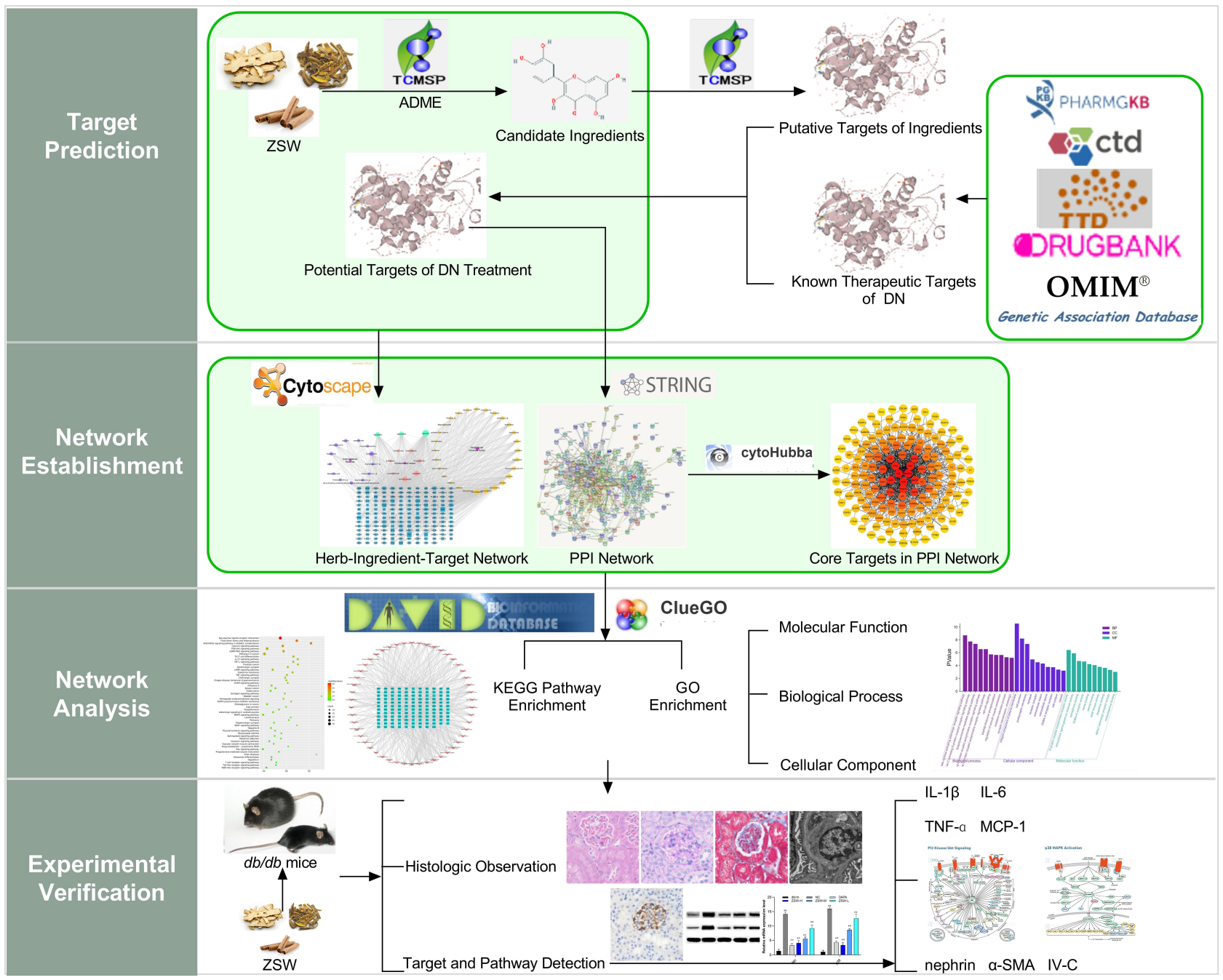

Figure I Flow diagram of network pharmacological analysis and experimental verification of ZSW for DN treatment.

Target Database (TTD, http://bidd.nus.edu.sg/group/cjttd/, updated on January 6,2020$),{ }^{19}$ DrugBank database (http:// www.drugbank.ca/,version 5.1.6, released on April 22, 2020), ${ }^{20}$ Online Mendelian Inheritance in Man (OMIM, http://www.omim.org/, updated on May 1, 2020) database, ${ }^{21}$ Genetic Association Database (GAD, https:// geneticassociationm.nih.gov/, updated on September 1, 2014), ${ }^{22}$ and Pharmacogenomics Knowledge Base (PHARMGKB, https://www.pharmgkb.org/, updated on April 28, 2020), ${ }^{23}$ using "diabetic nephropathy" as the search term. The type of target ID was unified to the Universal Protein Resource (UniProt, https://www. UniProt.org/, updated on October 15, 2019) database ID. $^{24}$ The potential targets in DN treatment were obtained by mapping the putative targets of the ingredients in ZSW and the known therapeutic targets in DN.

\section{Network Construction}

A "herb-ingredient-target" network was constructed and visualized with Cytoscape software (version 3.7.1, http:// www.cytoscape.org/, Boston, MA, USA) to explain the interactions between the candidate chemical ingredients of ZSW and their potential targets in DN treatment. ${ }^{25}$ Nodes represent herbs, candidate chemical ingredients and potential targets; edges represent interactions among herbs, candidate chemical ingredients and potential targets.

The protein-protein interaction (PPI) network of potential targets was constructed with the STRING (https://string-m.org, updated on January 19, 2019) database. ${ }^{26}$ The species was limited to human (Homo sapiens), and the confidence level was set at 0.700 . The results of PPI network construction were imported into Cytoscape software and analyzed with the cytoHubba plugin. The core targets in the PPI network were identified. 


\section{Biological Function and Pathway Analysis}

Gene ontology (GO) enrichment analysis mainly describes the biological functions of genes, such as their activity, process and site of action, and is widely used in gene function classification. Enrichment analysis of the biological process (BP), cellular component (CC) and molecular function (MF) terms associated with potential targets was performed with the ClueGO plugin of Cytoscape software. Kyoto Encyclopedia of Genes and Genomes (KEGG) pathway enrichment analysis was used to study signaling pathways related to genes with the Database for Annotation, Visualization and Integrated Discovery (DAVID, https://david.ncifcrf.gov/, updated on May 2016) Bioinformatics Resources 6.8 tool, with $P<$ $0.05 .^{27}$

\section{Experimental Validation}

\section{Animals}

Six-week-old male diabetic $\mathrm{db} / \mathrm{db}\left(\mathrm{C} 57 \mathrm{BLKS} / \mathrm{J}-\right.$ lepr $^{\mathrm{db}} /$ lepr $\left.^{\mathrm{db}}\right)$ mice and their male nondiabetic $\mathrm{db} / \mathrm{m}$ (C57BLKS/J-lepr ${ }^{\mathrm{db} /+}$ ) littermates were purchased from the Model Animal Research Center of Nanjing University (Nanjing, Jiangsu, China). The animals were housed under standard light (12 h light/dark cycle), temperature $\left(24 \pm 1{ }^{\circ} \mathrm{C}\right)$, and relative humidity (55 \pm $5 \%$ ) conditions and fed a pelletized commercial chow diet. The experiments were approved by Animal Experiments Ethics Committee of Beijing University of Chinese Medicine. All procedures were performed in accordance with the guidelines of the committee.

\section{Drugs and Reagents}

ZSW is composed of Anemarrhenae Rhizoma, Phellodendri Chinensis Cortex and Cinnamomum Cassia at a ratio of 20:20:3 according to the traditional experiences. Anemarrhenae Rhizoma and Phellodendri Chinensis Cortex were crushed for $30 \mathrm{~min}$, macerated in 8 volumes of ethanol $(50 \%, \mathrm{v} / \mathrm{v})$, and extracted two times for $60 \mathrm{~min}$ each. Cinnamomum Cassia was added to the filtered ethanol extracts and macerated in 8 volumes of ethanol $(50 \%, v / v)$. Reflux extraction was repeated twice. All ethanol extracts were combined and filtered. Last, the combined filtrate was concentrated using a rotary vacuum evaporator and was then lyophilized to obtain a powder. The yield of the ZSW extract was 11\%. Dapagliflozin (DAPA; $10 \mathrm{mg}$ tablets; AstraZeneca, Gaithersburg, MD, USA), a sodium-glucose cotransporter 2 (SGLT2) inhibitor that has been confirmed to have renoprotective and anti-inflammatory effects in diabetes mellitus models, ${ }^{28,29}$ was used as a positive control.

Primary antibodies against rabbit IL-1 $\beta$, IL-6, nephrin, phospho-p38 mitogen-activated protein kinase (p-p38 MAPK), $\alpha$-smooth muscle actin ( $\alpha$-SMA) and glyceraldehyde-3-phosphate dehydrogenase (GAPDH) as well as secondary antibodies were obtained from Abcam (Cambridge, UK). The primary antibody against rabbit MCP-1 was obtained from Proteintech (Chicago, IL, USA). The primary antibody against rabbit phospho-protein kinase B (p-Akt) was obtained from CST (Danvers, MA, USA). The primary antibody against rabbit TNF-a was obtained from GeneTeX (Irvine, CA, USA). The primary antibody against rabbit type IV collagen (IV-C) was obtained from Affinity Biosciences (Cincinnati, $\mathrm{OH}$, USA). Urinary albumin and neutrophil gelatinase-associated lipocalin (NGAL, lipocalin 2) enzyme-linked immunosorbent assay (ELISA) kits were obtained from Elabscience (Houston, TX, USA).

\section{Animal Model and Treatments}

After acclimation to the environment for 2 weeks, $d b / d b$ mice were randomly divided into 5 groups with 10 mice per group: the non-treated control (NC) group, high-dose ZSW (ZSW-H, $6.0 \mathrm{~g} / \mathrm{kg}$ ) group, medium-dose ZSW (ZSW-M, $3.0 \mathrm{~g} / \mathrm{kg}$ ) group, low-dose ZSW (ZSW-L, 1.5 $\mathrm{g} / \mathrm{kg})$ group and DAPA $(1.0 \mathrm{mg} / \mathrm{kg})$ group. ZSW and DAPA were dissolved in deionized water for oral gavage once daily at the doses mentioned above. Mice in NC group and $\mathrm{db} / \mathrm{m}$ mice received only an equivalent volume of deionized water over the same treatment period. Mice had access to food and water ad libitum. The treatment duration was 12 weeks.

\section{Sample Collection}

Samples for fasting blood glucose (FBG) measurement were collected by tail vein bleeding, and FBG was measured every 2 weeks. Mice were housed in individual metabolic cages for urine sample collection every 4 weeks. At the end of the 12th week of treatment, blood samples were collected by retro-orbital bleeding, and all mice were then euthanized and sacrificed. Renal tissue samples were harvested and divided into two parts. One part was fixed with $4 \%$ paraformaldehyde and stored at $4{ }^{\circ}$ $\mathrm{C}$ for section preparation, and the other part was stored at $-80{ }^{\circ} \mathrm{C}$ for Western blotting (WB) and real-time quantitative polymerase chain reaction (RT-qPCR) analyses. 


\section{Blood and Urine Examination}

Serum and urinary creatinine (CRE) and blood urea nitrogen (BUN) were measured in an automatic biochemical analyzer (Xinrui, Zhongshan, Guangdong, China). The urinary albumin concentration and urinary NGAL were measured with ELISA kits (Elabscience, Houston, TX, USA) in accordance with the manufacturer's instructions. Urinary albumin excretion was evaluated by the ratio of the urinary albumin concentration to the CRE concentration (ACR, $\mu \mathrm{g} / \mathrm{mg}$ ).

\section{Renal Histologic Analysis}

Renal tissue samples fixed with $4 \%$ paraformaldehyde were dehydrated through an alcohol concentration gradient and were then cleared in xylene, embedded in paraffin, and finally sliced into $3 \mu \mathrm{m}$ thick sections. The sections were stained with hematoxylin and eosin (HE), periodic acidSchiff (PAS) and Masson trichrome and were then examined by light microscope (Olympus, Shinjuku, Japan) at $400 \times$ magnification for histopathological diagnosis. Percentage of glomerular collagenous fiber was analyzed using Image-Pro Plus 6.0 (Media Cybernetics, Inc., Rockville, MD, USA). ${ }^{30}$ Tubulointerstitial damage index were calculated according to reference. ${ }^{31}$ For transmission electron microscopy (TEM) observation and GBM thickness measurement, renal cortical tissues were sectioned at $1 \mathrm{~mm}$, fixed with $3 \%$ glutaraldehyde, postfixed in $1 \%$ osmium tetroxide, dehydrated through a graded alcohol series and embedded in Epon 812 epoxy. Ultrathin sections $(60 \mathrm{~nm})$ were sliced with an ultramicrotome (RMCBoeckeler Instruments, Tucson, AZ, USA) and were then stained with uranyl acetate and lead citrate. The sections were examined under an electron microscope (FEI Tecnai Spirit, Dayton, OH, USA) at $6000 \times$ magnification.

\section{Immunohistochemical (IHC) Analysis}

Sections $(3 \mu \mathrm{m})$ of paraffin-embedded renal tissue were deparaffinized and rehydrated and were then incubated with $3 \% \mathrm{H}_{2} \mathrm{O}_{2}$ for 10 min to quench endogenous peroxidase activity. The sections were then incubated overnight at $4{ }^{\circ} \mathrm{C}$ with diluted primary antibodies against nephrin, IV-C and $\alpha$-SMA (1:4000 dilution) respectively. Images were analyzed using Image-Pro Plus 6.0 software, and staining values were expressed as the positive staining rates.

\section{WB Analysis}

Total protein from renal tissues was extracted with icecold RIPA lysis buffer (Beyotime, Shanghai, China). The lysate was centrifuged at $10,000 \times \mathrm{g}$ for $15 \mathrm{~min}$ at $4{ }^{\circ} \mathrm{C}$. The protein concentrations were determined with a $\mathrm{BCA}$ Protein Assay Kit (Solarbio, Beijing, China). Equal amounts of protein were separated by SDS-PAGE and transferred to PVDF membranes (Millipore, Burlington, MA, USA). Membranes were blocked in 5\% nonfat milk at room temperature for $1 \mathrm{~h}$ and were then incubated with primary antibodies against IL-1 $\beta$, IL-6, nephrin, p-Akt (all at a 1:1000 dilution), IV-C, MCP-1, p-p38 MAPK, TNF-a (all at a 1:500 dilution), $\alpha$-SMA (1:100 dilution) and GAPDH (1:5000 dilution) respectively overnight at $4{ }^{\circ} \mathrm{C}$ and were then washed. After incubation with the appropriate secondary antibodies (1:1000 dilution) for $1 \mathrm{~h}$ at room temperature, target protein bands were detected with enhanced chemiluminescence substrate (Solarbio, Beijing, China). Each WB analysis was performed three times. Semiquantitative analysis was performed using ImageJ software (National Institutes of Health, Bethesda, MD, USA).

\section{RT-qPCR Analysis}

Total RNA from renal tissues was extracted using TRIzol reagent (Invitrogen, Carlsbad, CA, USA) according to the manufacturer's instructions. RNA $(0.2 \mu \mathrm{g})$ was reverse transcribed into cDNA using a SuperScript III reverse transcription reagent kit (Invitrogen, Carlsbad, CA, USA), and equal amounts of the reverse transcription products were subjected to PCR amplification. Real-time PCR was performed in a StepOne Software Real-Time PCR system (Applied Biosystems, Foster City, CA, USA). $\beta$-Actin was amplified in a parallel reaction as an internal quantitative control. The thermal cycling program used for PCR was as follows: $95^{\circ} \mathrm{C}$ for $10 \mathrm{~s}$, followed by 40 cycles at $58{ }^{\circ} \mathrm{C}$ for $20 \mathrm{~s}, 72{ }^{\circ} \mathrm{C}$ for $20 \mathrm{~s}$ and $95{ }^{\circ} \mathrm{C}$ for 5 min. The amplification curve and melting curve for realtime fluorescence quantitative PCR were performed at the end of the reaction. The cycle threshold $(\mathrm{Ct})$ values were obtained for each sample well. The $\mathrm{Ct}$ value of $\beta$-actin in the same sample was taken as the internal normalization parameter, and relative quantitative expression levels were calculated by the $2^{-\Delta \Delta \mathrm{Ct}}$ method. Each group was analyzed in triplicate. The primer sequences used for mouse mRNA detection are listed in Table 1.

\section{Statistical Analysis}

All data are shown as the mean \pm standard deviation (SD) values. Data were analyzed with Statistical Product and Service Solutions software 22.0 (Chicago, IL, USA). 
Table I Primers Sequences of RT-qPCR

\begin{tabular}{|l|l|l|}
\hline Target & Forward $\mathbf{( 5 '}$ to $\mathbf{3}^{\prime} \mathbf{)}$ & Reverse $\mathbf{( \mathbf { 5 } ^ { \prime }}$ to $\mathbf{3}^{\prime} \mathbf{)}$ \\
\hline IV-C & TGAACAAGCTCTGGAGTGGGTACAG & GTACAGGAAAGGCATGGTGCTGAAG \\
$\alpha$-SMA & GGAAAAGATCTGGCACCACTC & TTTCTCCCGGTTGGCCTT \\
Akt & ATGAACGACGTAGCCATTGTG & TTGTAGCCAATAAAGGTGCCAT \\
IL-I $\beta$ & GTGAAATGCCACCTTTTGACA & GATTTGAAGCTGGATGCTCT \\
IL-6 & CTTCCATCCAGTTGCCTT & CTGTGAAGTCTCCTCTCCG \\
MCP-I & CTGCTGCTACTCATTCACCA & CTTCTTTGGGACACCTGC \\
nephrin & TCTGGGTCCAAACCCTAAGATT & TCAATAAGCAGGTGGAACTCAC \\
P38 MAPK & TGACCCTTATGACCAGTCCTTT & GTCAGGCTCTTCCACTCATCTAT \\
TNF- $\alpha$ & TTCCCAAATGGCCTCCCTC & TCCAGCTGCTCCTCCACT \\
$\beta-A c t i n$ & CCCATCTATGAGGGTTACGC & TTTAATGTCACGCACGATTTC \\
\hline
\end{tabular}

Analysis of variance (ANOVA) was carried out to determine statistical significance for comparisons among multiple parameters. The least significant difference (LSD) test was performed under the assumption of equal variances; otherwise, Dunnett's T3 test was applied for data with unequal variances. Statistical significance was established at $P<0.05$ or $P<0.01$.

\section{Results}

\section{Chemical Ingredients in the Candidates}

A total of 56 chemical ingredients in the three herbal medicines in ZSW were screened in TCMSP by ADME parameters $(\mathrm{OB} \geq 30 \%$ and $\mathrm{DL} \geq 0.18)$ : 15 ingredients in Anemarrhenae Rhizoma, 36 ingredients in Phellodendri Chinensis Cortex and 10 ingredients in Cinnamomum Cassia. Among the screened compounds, stigmasterol was the common constituent of the three herbal medicines. Kaempferol was found in both Anemarrhenae Rhizoma and Cinnamomum Cassia, while quercetin was found in both Phellodendri Chinensis Cortex and Cinnamomum Cassia.

\section{Prediction of Potential Targets}

The putative targets of the candidate ingredients in ZSW were identified in the TCMSP database: 94 for Anemarrhenae Rhizoma, 137 for Phellodendri Chinensis Cortex and 142 for Cinnamomum Cassia. In total, 18,824 known therapeutic targets for DN were identified in CTD, TTD, DrugBank, OMIM, GAD and PHARMGKB. Finally, 166 potential treatment targets in $\mathrm{DN}$ were obtained.

\section{Network Construction}

The "herb-ingredient-target" interaction network of ZSW for DN treatment was constructed with Cytoscape software and contained 220 nodes and 1093 edges shown in Figure 2. The average degree value in the network was 9.93. The top 20 candidate chemical ingredients by degree value were listed in Table 2.

The PPI network was constructed with STRING database and Cytoscape cytoHubba plugin with a confidence level of 0.700 . A total of 154 targets were screened and analyzed as shown in Figure 3. The average degree value in the PPI network was calculated to be 10.59. Sixty selected core targets had a degree value larger than the average value. Among the top 20 core targets listed in Table 3, IL6, IL1B, TNF and CCL2 were coding genes of inflammatory cytokines, targets coded by SAA1, ESR1, AR, F2 were closely related to inflammatory response, MAPK1, MAPK8, MAPK3 and MAPK14 were coding genes of MAPK family participating in inflammation.

\section{Biological Function and Pathway Analysis}

Biological function analysis of the candidate chemical ingredients in $\mathrm{ZSW}$ for $\mathrm{DN}$ treatment was carried out with the Cytoscape ClueGO plugin, with $P<0.05$. The key targets were involved in biological processes such as the G-protein coupled receptor signaling pathway, lipopolysaccharide-mediated signaling pathway, oxidationreduction process, synaptic transmission, and cell-cell signaling as shown in Figure 4A. GO cell component enrichment analysis indicated that the targets were enriched mainly in the terms cell junction, synapse, ECM, and cytosol shown in Figure 4B. The enriched molecular function terms were mainly G-protein coupled receptor activity, neurotransmitter binding, and activation of MAPK activity, as shown in Figure 4C. The GO enrichment results for the top 10 terms are shown as a histogram in Figure 4D. 


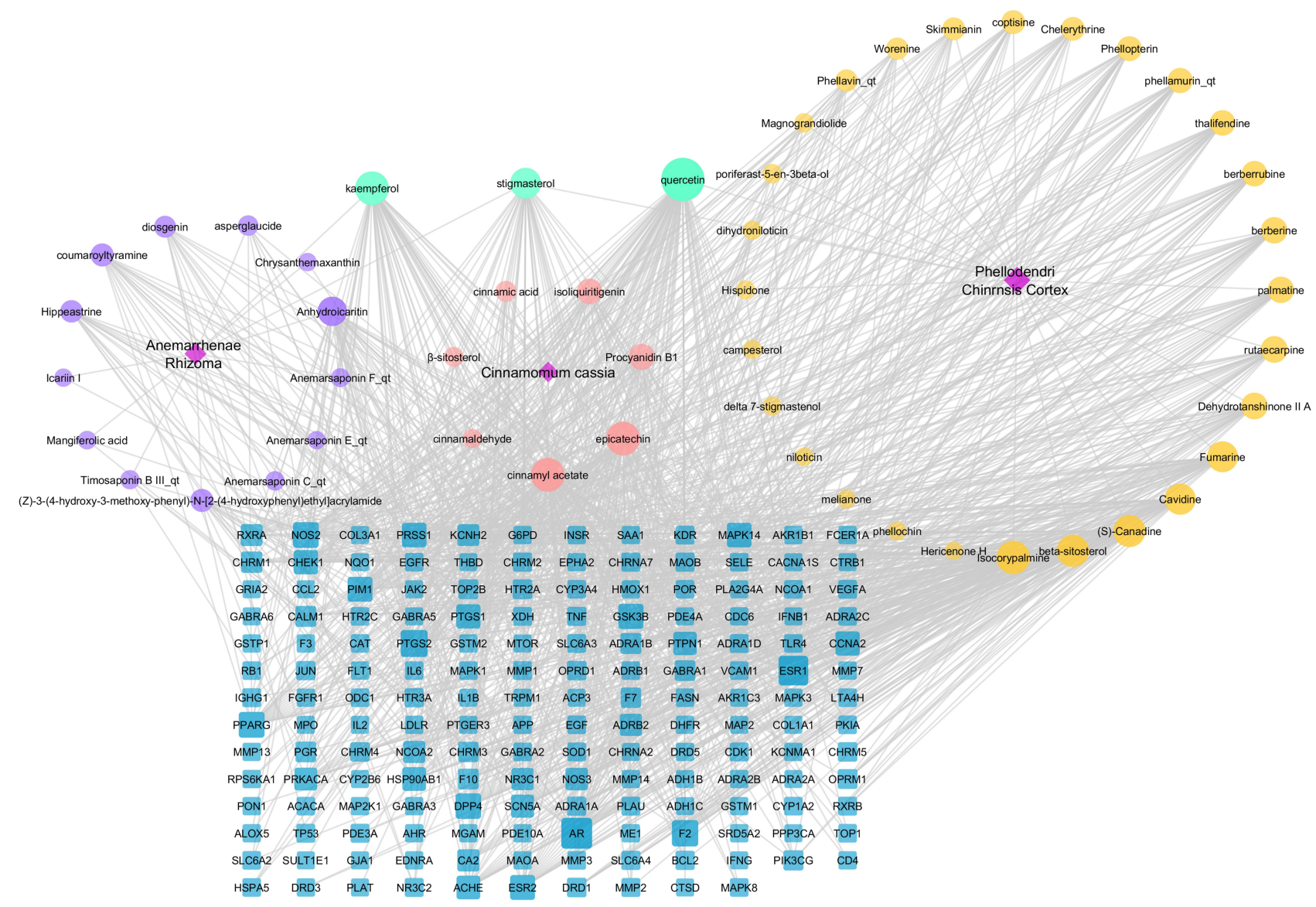

Figure 2 The "herb-ingredient-target" interaction network of ZSW for DN treatment by pharmacological analysis. The purple part represents the unique active components of Anemarrhenae Rhizoma. The yellow part represents the unique active components of Phellodendri Chinensis Cortex. The pink part represents the unique active components of Cinnamomum Cassia. The blue nodes represent the target of chemical composition interaction. The gray edge represents the interaction between compound molecule and target.

Pathway enrichment analysis of ZSW for DN treatment was performed with DAVID Bioinformatics Resources 6.8 tool, and 167 significant pathways were identified as those with $P<0.05$. The enrichment results of the top $50 \mathrm{KEGG}$ pathways are shown in Figure 5A by a bubble chart. The pathways included inflammatory reaction, advanced glycosylation end products (AGEs), cell proliferation and differentiation, endocrine resistance, nerve tissue conduction and so on. Considerable quantities of pathways related to inflammatory response such as PI3K-Akt signaling pathway, IL-17 signaling pathway, MAPK signaling pathway, $\mathrm{T}$ cell receptor signaling pathway, Toll-like receptor signaling pathway, and NOD-like receptor signaling pathway existed in top 50 pathways. As shown in the "target-top 50 pathways" network constructed by Cytoscape software (Figure 5B), PI3K-Akt signaling pathway and MAPK signaling pathway contained the largest related targets.

\section{ZSW Improved the Levels of Blood Sugar and Renal Injury Biochemical Index in $\mathrm{db} / \mathrm{db}$ Mice}

The hypoglycemic effect of ZSW has been confirmed by previous studies. As expected, our study showed that FBG levels were much higher in eight-week-old $\mathrm{db} / \mathrm{db}$ mice than in $\mathrm{db} / \mathrm{m}$ mice of the same age $(P<0.01)$. Biweekly blood glucose tests showed that as early as the second week of treatment, all doses of ZSW and DAPA significantly decreased FBG levels in $\mathrm{db} / \mathrm{db}$ mice $(P<0.01)$. These results are shown in Figure 6A. The renoprotective effect of ZSW as a TCM compound in $\mathrm{db} / \mathrm{db}$ mice had not been explored in the past. As shown in Figure 6B and $C$, compared to that of $\mathrm{db} / \mathrm{m}$ mice, both urinary ACR and NGAL levels of $\mathrm{db} / \mathrm{db}$ mice were increased, and were effectively reduced by ZSW and DAPA treatment $(P<0.01)$. As shown in Figure 6D and E, both the BUN and serum CRE 
Table 2 General Information of the Top 20 Ingredients in Network of ZSW in Treating DN

\begin{tabular}{|c|c|c|c|c|c|}
\hline Mol ID & Ingredient & Herb & OB (\%) & DL & Degree \\
\hline MOL000098 & Quercetin & P. C. Cortex, C. Cassia & 46.3 & 0.28 & 86 \\
\hline MOL00682I & Epicatechin & C. Cassia & 55.09 & 0.77 & 54 \\
\hline MOL000422 & Kaempferol & A. Rhizoma, C. Cassia & 41.88 & 0.24 & 54 \\
\hline MOL003526 & Cinnamyl acetate & C. Cassia & 21.15 & 0.04 & 54 \\
\hline MOL000790 & Isocorypalmine & P. C. Cortex & 35.77 & 0.59 & 53 \\
\hline MOL000358 & Beta-sitosterol & P. C. Cortex & 36.91 & 0.75 & 49 \\
\hline MOL00I455 & (S)-Canadine & P. C. Cortex & 53.83 & 0.77 & 48 \\
\hline MOL002670 & Cavidine & P. C. Cortex & 35.64 & 0.81 & 45 \\
\hline MOL000449 & Stigmasterol & A. Rhizoma, P. C. Cortex, C. Cassia & 43.83 & 0.76 & 44 \\
\hline MOL000787 & Fumarine & P. C. Cortex & 59.26 & 0.83 & 44 \\
\hline MOL004373 & Anhydroicaritin & A. Rhizoma & $45.4 I$ & 0.44 & 39 \\
\hline MOL00265I & Dehydrotanshinone II A & P. C. Cortex & 43.76 & 0.4 & 30 \\
\hline MOL002662 & Rutaecarpine & P. C. Cortex & 40.3 & 0.6 & 30 \\
\hline MOL00I454 & Berberine & P. C. Cortex & 38.86 & 0.78 & 29 \\
\hline MOL000785 & Palmatine & P. C. Cortex & 64.6 & 0.65 & 29 \\
\hline MOL000004 & Procyanidin BI & C. Cassia & 67.87 & 0.66 & 28 \\
\hline MOL002894 & Berberrubine & P. C. Cortex & 35.74 & 0.73 & 26 \\
\hline MOL006422 & Thalifendine & P. C. Cortex & $44.4 I$ & 0.73 & 26 \\
\hline MOL00II3I & Phellamurin_qt & P. C. Cortex & 56.6 & 0.39 & 25 \\
\hline MOL002644 & Phellopterin & P. C. Cortex & 40.19 & 0.28 & 24 \\
\hline
\end{tabular}

Notes: The top 20 ingredients in network of ZSW in treating DN screened via Cytoscape software were sorted by degree. The Mol ID, OB, and DL of the ingredients were obtained by screening TCMSP database.

Abbreviations: A. Rhizoma, Anemarrhenae Rhizoma; P. C. Cortex, Phellodendri Chinensis Cortex; C. Cassia, Cinnamomum Cassia; OB, oral bioavailability; DL, drug similarity.

levels were higher in $\mathrm{db} / \mathrm{db}$ mice than in $\mathrm{db} / \mathrm{m}$ mice $(P<0.01)$.

DAPA and all doses of ZSW significantly blunted the increases in BUN and serum CRE $(P<0.01)$. All these results demonstrated that ZSW improved the levels of blood sugar and renal injury biochemical index in $\mathrm{db} / \mathrm{db}$ mice.

\section{ZSW Ameliorated Pathological Damage and Regulated Related Indicators in $\mathrm{db} / \mathrm{db}$ Mice}

Pathological changes can directly show the therapeutic effects. In the present study, HE, PAS and Masson staining as well as TEM observation showed glomerular hypertrophy, GBM thickening, mesangial matrix expansion and tubular inflammation in $\mathrm{db} / \mathrm{db}$ mice. However, these injuries were attenuated to varying extents by ZSW and DAPA treatment, as shown in Figure 7. Moreover, as shown in Figure 7B-D, the percentage of glomerular collagenous fiber, GBM thickness, and tubulointerstitial damage index were remarkably higher in $\mathrm{db} / \mathrm{db}$ mice than in $\mathrm{db} / \mathrm{m}$ mice $(P<0.01)$. DAPA and all doses of ZSW significantly decreases all these three pathological quantitative indicators $(P<0.01)$.
We further explored the effect of ZSW on markers of renal proper cells and indicators of pathological injuries. As a marker of podocytes, nephrin expression was markedly lower in the renal tissue of $\mathrm{db} / \mathrm{db}$ mice than in the renal tissue of $\mathrm{db} / \mathrm{m}$ mice. This reduction in nephrin expression was reversed by ZSW and DAPA treatment. The increased IV-C level in $\mathrm{db} / \mathrm{db}$ mice indicated ECM proliferation in renal tissues, and the IV-C level was decreased by ZSW and DAPA treatment. The expression level of $\alpha$-SMA, a marker of fibrosis, was higher in $\mathrm{db} / \mathrm{db}$ mice than in $\mathrm{db} / \mathrm{m}$ mice, and $\alpha$-SMA expression was inhibited by DAPA and ZSW treatment. All these findings were determined by IHC analysis $(P<0.01)$, as shown in Figure 8 . The protein and mRNA levels of these biomarkers were subsequently measured by WB analysis and RTqPCR $(P<0.01)$, as shown in Figure 9.

\section{ZSW Inhibited the Expression of Multiple Inflammatory Cytokines in $\mathrm{db} / \mathrm{db}$ Mice}

According to the result of core genes screened by PPI and cytoHubba analysis, we chose inflammatory cytokines including IL-1 $\beta$, IL-6, TNF- $\alpha$, and MCP-1 for experimental validation. As shown in Figure 10, the 


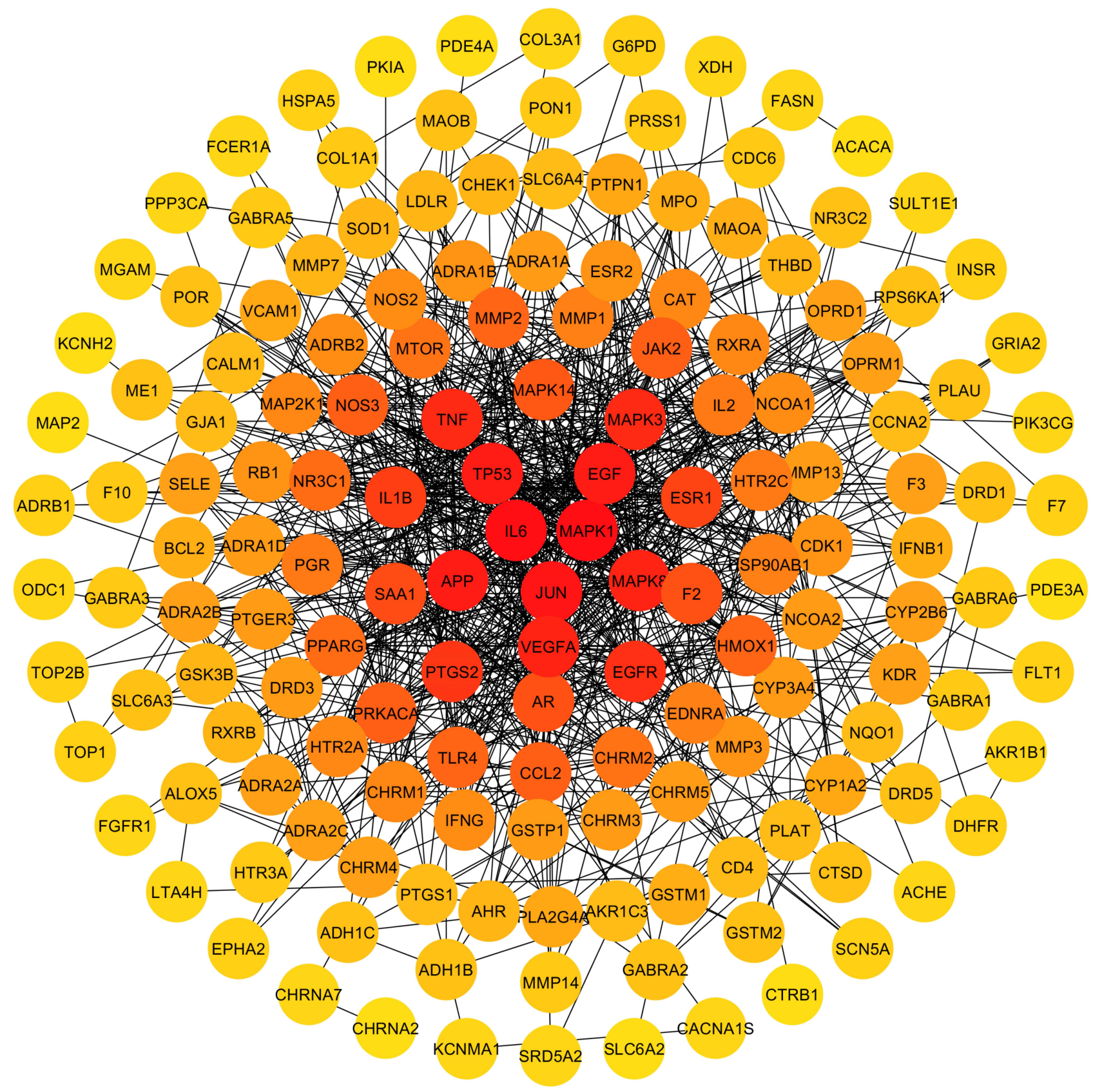

Figure 3 The PPI network map of target in ZSW for DN treatment. The spot represents the potential target of DN treatment. The darker spot closer to the center has higher degree value. The black edge represents the interaction between targets.

protein levels of inflammatory cytokines including IL-1 $\beta$, IL-6, TNF- $\alpha$, and MCP-1 were significantly increased in the NC group compared with those in the $\mathrm{db} / \mathrm{m}$ group $(P<0.01)$, as were the mRNA levels of these cytokines $(P<0.01)$. The protein and mRNA levels of the abovementioned inflammatory cytokines were significantly reduced in $\mathrm{db} / \mathrm{db}$ mice treated with ZSW or DAPA compared with $\mathrm{NC}$ mice $(P<0.01)$.

\section{ZSW Regulated the PI3K-Akt and p38 MAPK Signaling Pathways in $\mathrm{db} / \mathrm{db}$ Mice}

Considering the importance in the "target-top 50 pathways" network analyzed in the present study, PI3K-Akt and p38 MAPK signaling pathways were selected for experimental validation as major inflammation-related signaling pathways. As shown in Figure 11, the levels of p-Akt and p-p38 were significantly increased in $d b / d b$ 
Table 3 The Top 20 Core Targets in PPI Network of ZSW in Treating DN

\begin{tabular}{|l|l|l|}
\hline Gene & Target & Degree \\
\hline IL6 & Interleukin-6 & $4 I$ \\
MAPKI & Mitogen-activated protein kinase I & $4 I$ \\
JUN & Transcription factor AP-I & 40 \\
APP & Amyloid-beta precursor protein & 38 \\
TP53 & Cellular tumor antigen p53 & 38 \\
EGF & Pro-epidermal growth factor & 38 \\
MAPK8 & Mitogen-activated protein kinase 8 & 37 \\
VEGFA & Vascular endothelial growth factor A & 37 \\
TNF & Tumor necrosis factor & 33 \\
MAPK3 & Mitogen-activated protein kinase 3 & 33 \\
EGFR & Epidermal growth factor receptor & 32 \\
PTGS2 & Prostaglandin G/H synthase 2 & 28 \\
ILIB & Interleukin-I beta & 27 \\
ESRI & Estrogen receptor & 26 \\
SAAI & Serum amyloid A-I protein & 24 \\
F2 & Prothrombin & 22 \\
AR & Androgen receptor & 22 \\
MAPKI4 & Mitogen-activated protein kinase 14 & 21 \\
CCL2 & C-C motif chemokine 2 & 20 \\
NOS3 & Nitric oxide synthase, endothelial & 20 \\
\hline
\end{tabular}

Note: The top 20 core targets in PPI network of ZSW in treating DN constructed by STRING database were screened via Cytoscape cytoHubba plugin and sorted by degree.

mice compared with $\mathrm{db} / \mathrm{m}$ mice $(P<0.01)$. Moreover, compared with those in $\mathrm{db} / \mathrm{db}$ mice, the levels of $\mathrm{p}$-Akt and p-p38 were significantly decreased by 12 weeks of ZSW or DAPA treatment $(P<0.01)$. The mRNA levels of Akt and p38 were increased in $\mathrm{db} / \mathrm{db}$ mice compared with $\mathrm{db} / \mathrm{m}$ mice $(P<0.01)$ and were significantly lower in all ZSW groups and the DAPA group than in the NC group $(P<0.01)$. All these findings declared that $\mathrm{ZSW}$ regulated the PI3K-Akt and p38 MAPK signaling pathways in $\mathrm{db} / \mathrm{db}$ mice.

\section{Discussion}

Abnormal glycolipid metabolism, inflammation, oxidative stress, AGEs, hemodynamic disorders and other factors are implicated in DN pathogenesis. ${ }^{32}$ Although previous studies have confirmed that some of the main active ingredients of ZSW have hypoglycemic and renal protective effects, ${ }^{33-35}$ while cannot fully reflect the multi-target and multi-pathway activities therapeutic characteristics and related mechanisms of ZSW as a TCM compound. In the present study, the bioactive compounds and the molecular mechanisms of ZSW for DN therapy were firstly revealed by a network pharmacology-based approach. The abovementioned mechanisms were validated in the $\mathrm{db} / \mathrm{db}$ mouse model as the main mechanistic targets of ZSW in DN therapy. A remarkable number of core targets and signaling pathways were screened by PPI network and KEGG pathway enrichment analysis in this study, rising a significant research direction of ZSW for DN treatment.

Chronic low-grade inflammation exists widely in patients with diabetes mellitus and is essential for the progression of DN. ${ }^{36}$ Notably, inflammation is attributed to glucose metabolism disorder, which leads to glucose self-oxidation and metabolic stress responses. ${ }^{37}$ Consequently, blood glucose control is the basic principle in the prevention and treatment of DN. As a model of spontaneous diabetes, $\mathrm{db} / \mathrm{db}$ mice exhibit increased blood glucose levels as a result of leptin receptor gene mutation and functional loss. ${ }^{38}$ Our study demonstrated that ZSW exerted a hypoglycemic effect, which might be an underlying renoprotective and anti-inflammatory mechanism.

As they mimic many of the characteristics of early human $\mathrm{DN}, \mathrm{db} / \mathrm{db}$ mice are widely used in efficacy and mechanistic studies of DN therapies. ${ }^{39}$ Microalbuminuria can be detected in the early stage, as well as increase of other early renal injury markers, such as NGAL which reflects tubular injury. ${ }^{40}$ As DN progresses, increasing proteinuria is the major character, and eventually leads to renal dysfunction. Regarding histopathological features, the glomerular hypertrophy, GBM thickening, mesangial expansion and matrix deposition exist in $\mathrm{db} / \mathrm{db}$ mice. In addition, the extent of tubulointerstitial injury shown as vacuolar degeneration and inflammatory cell infiltration ultimately determines the rate of attrition of renal function. ${ }^{41}$ The typical marker proteins molecularly reflect variation of both intrinsic renal cells and pathological processes. Nephrin maintains the functions of the renal filtration barrier. Previous studies have shown that high glucose reduces the stability of nephrin, which often leads to podocyte injury, proteinuria and renal failure. ${ }^{42} \mathrm{IV}-\mathrm{C}$ is physiologically expressed in the GBM. However, excessive IV-C secretion leads to the accumulation of ECM, resulting in GBM thickening and mesangial matrix expansion in $\mathrm{DN},{ }^{43}$ The number of myofibroblasts is inversely correlated with renal function in DN. $\alpha$-SMA is the major cytoskeletal protein expressed by myofibroblasts as activated fibroblasts and closely related to progressive renal fibrosis in DN. ${ }^{44} \alpha$-SMA is expressed in cells primarily located in the renal interstitium and, to a lesser extent, in glomeruli in association with mesangial proliferation. ${ }^{45}$ In this study, ZSW improved indexes of renal injury, 


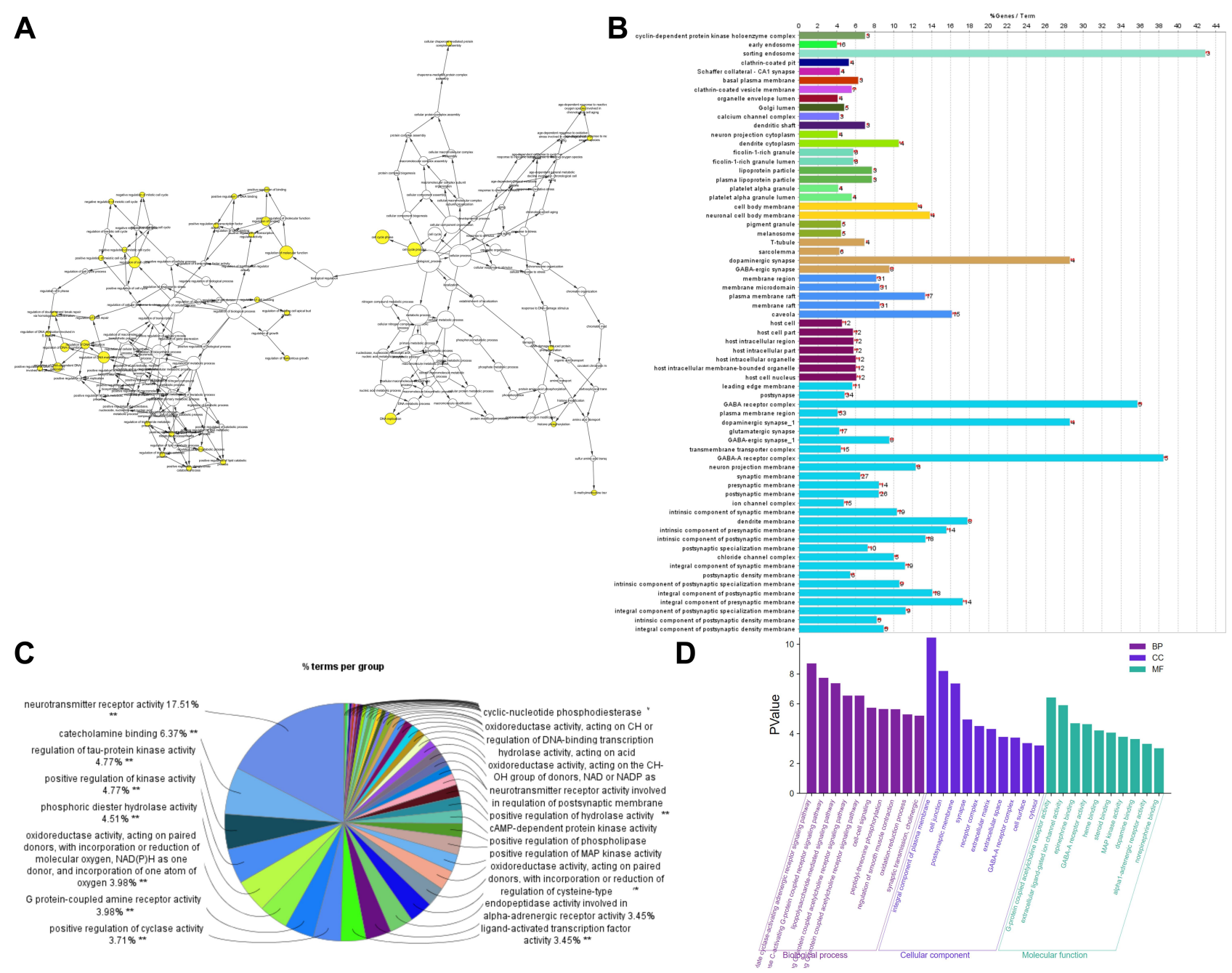

Figure 4 GO enrichment analysis of target in ZSW for DN treatment. (A) GO biological process enrichment analysis; (B) GO cell component enrichment analysis; (C) GO molecular function enrichment analysis; (D) the top $10 \mathrm{GO}$ enrichment results.

ameliorated pathological damage and regulated related indicators in $\mathrm{db} / \mathrm{db}$ mice, suggesting the renoprotective effects of ZSW in DN treatment.

Several inflammation-related genes including IL6, IL1B, TNF and CCL2 were listed in the top 20 core targets with high degree by PPI network analysis, providing a strong reason for index selection of further research. Cytokines are essential for mediating inflammation throughout the progression of DN. ILs are classical inflammatory factors. IL-6, encoded by the IL6 gene, promotes ECM production and mesangial cell proliferation, changes vascular permeability, and accelerates GBM thickening. IL-1 $\beta$, encoded by the IL1B gene, activates and aggregates immune cells; induces the synthesis and release of other inflammatory cytokines (such as IL-6), chemokines and adhesion molecules; and amplifies the local or systemic inflammatory response. TNF- $\alpha$, encoded by the TNF gene, promotes renal cell apoptosis and microalbuminuria, and accelerates renal fibrosis. A previous study found that, compared with patients free of renal injury, the level of serum TNF- $\alpha$ significantly increased in DN patients. ${ }^{46}$ MCP-1, which is encoded by CCL2, is the most wellknown chemokine regulator of monocytes and is upregulated in DN patients. It regulates the expression of adhesion molecules, increases and recruits monocytes to the glomerulus. Moreover, it promotes the release of inflammatory mediators such as IL-1 and IL-6, induces oxidative stress, activates proteolytic enzymes and damages endothelial cells. ${ }^{47}$ We investigated the abovementioned cytokines as the core targets of ZSW in DN therapy by network pharmacology analysis and made an experimental verification in vivo, suggesting a potential mechanism of renal protection in DN treatment. 


\section{A}

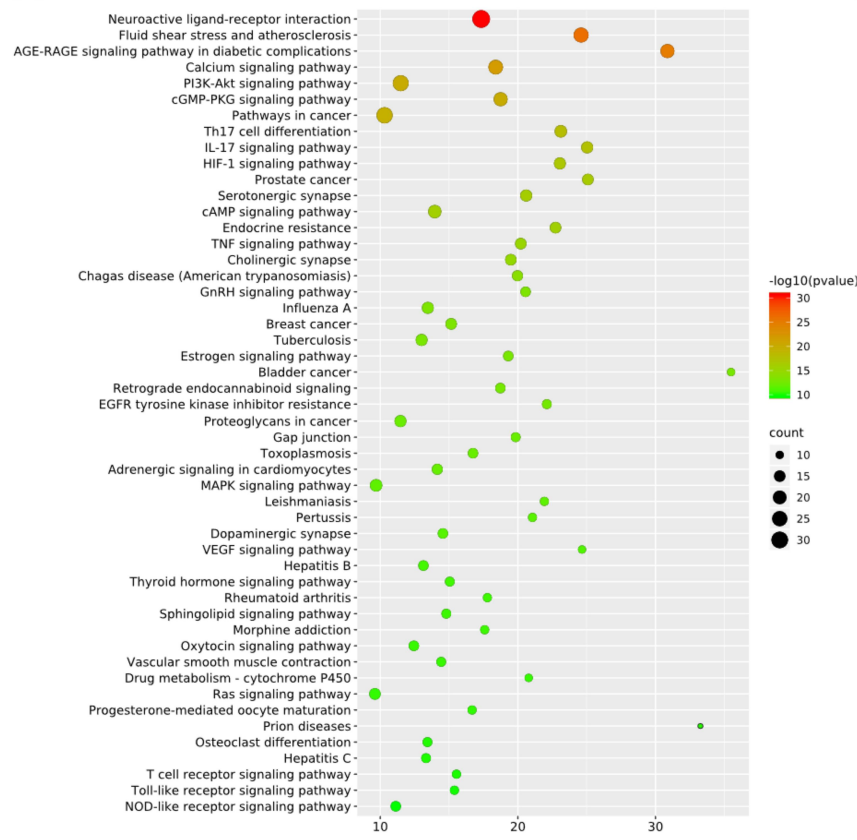

B

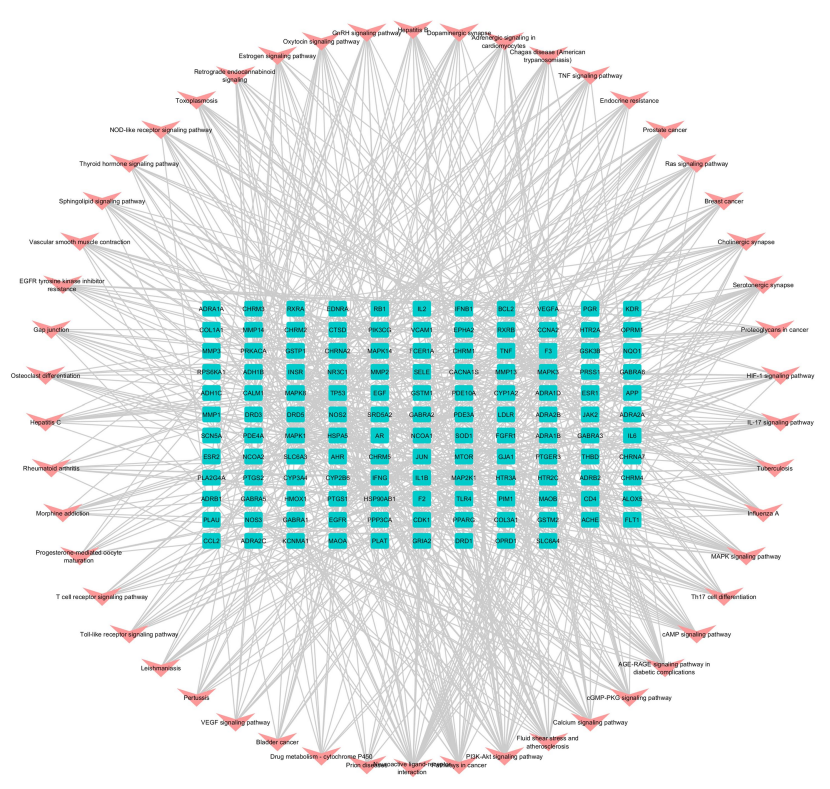

Figure 5 KEGG pathway enrichment analysis of target in ZSW for DN treatment. (A) The enrichment results of the top 50 KEGG pathways. (B) The "targets-top 50 pathways" network. The rose-red arrow represents the pathway. The green rectangle represents the potential target of DN treatment. The gray edge represents the interaction between chemical ingredient and target.

A

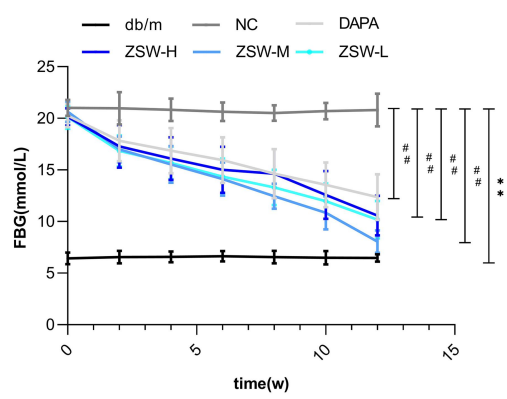

B

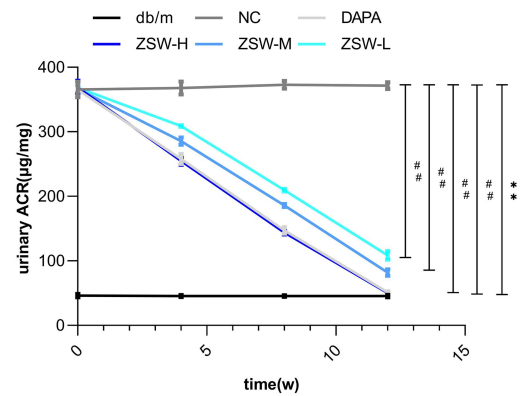

C

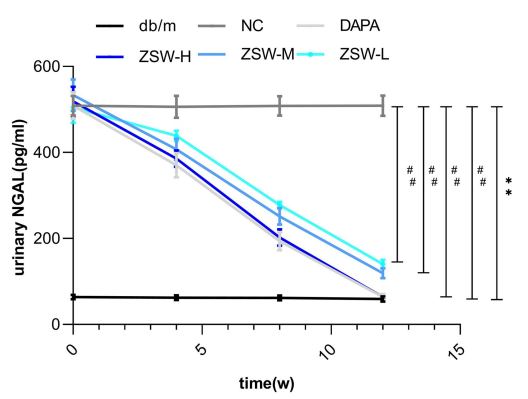

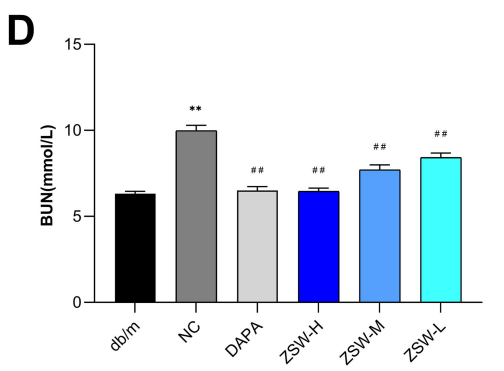

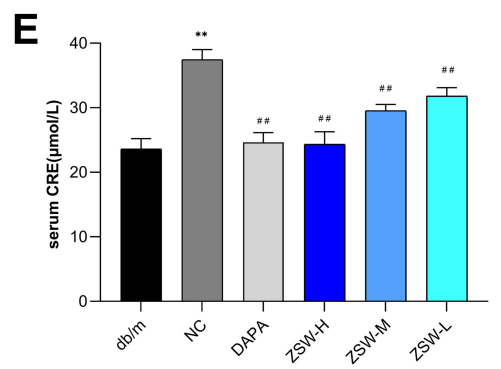

Figure 6 ZSW treatment alleviates levels of FBG (A) and major biochemical indexes of renal injuries including urinary ACR (B), urinary NGAL(C), BUN (D) and serum CRE $(E)$ in $\mathrm{db} / \mathrm{db}$ mice. Data are presented as mean $\pm \mathrm{SD}(\mathrm{n}=10) . * * P<0.0 \mathrm{l}$, vs $\mathrm{db} / \mathrm{m}$ group; ${ }^{\# \#} P<0.0 \mathrm{I}$, vs $\mathrm{NC}$ group.

As a result of higher $P$ value and more targets related to shown by KEGG pathway enrichment analysis, PI3K-Akt and MAPK signaling pathway were selected for experimental validation. MAPK is a member of the serine/threonine protein kinase family. ${ }^{48}$ Among the four members of the MAPK family, p38 MAPK plays a critical role in inflammation. Once activated by inflammation-related cytokines, it promotes cell proliferation and ECM production in DN. ${ }^{49}$ The PI3K-Akt signaling pathway mediates the 


\section{A}
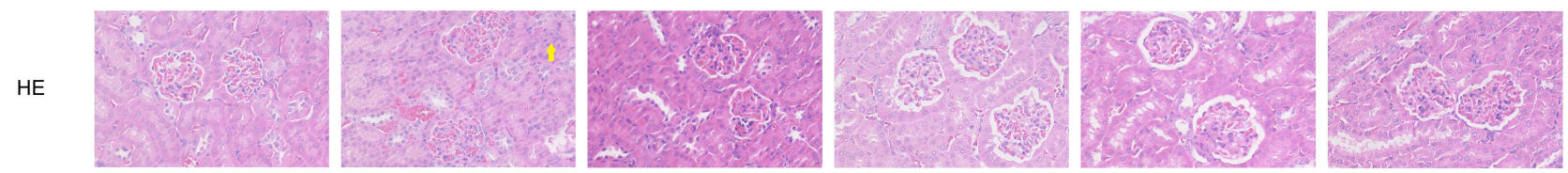

PAS
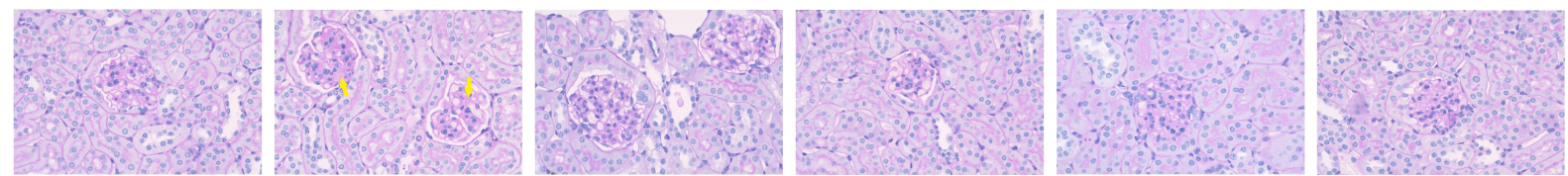

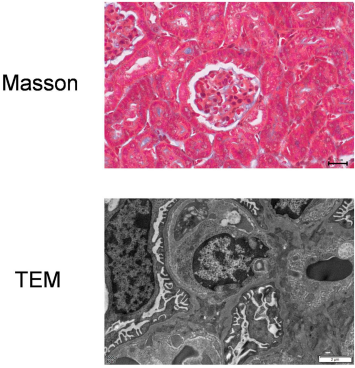

$\mathrm{db} / \mathrm{m}$

B

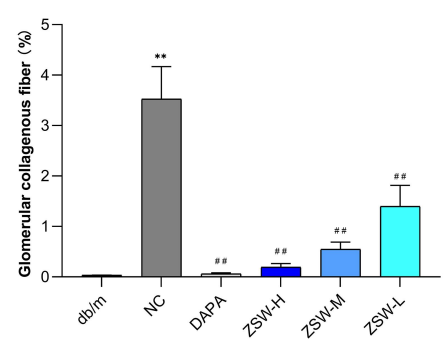

NC
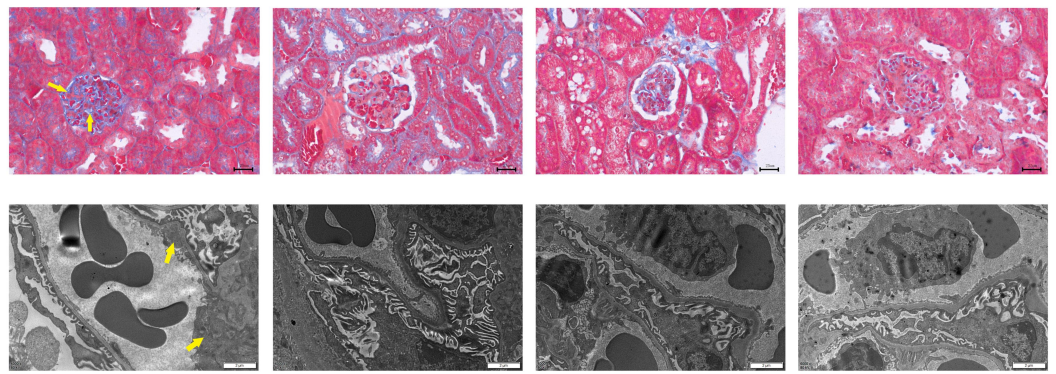

DAPA

C

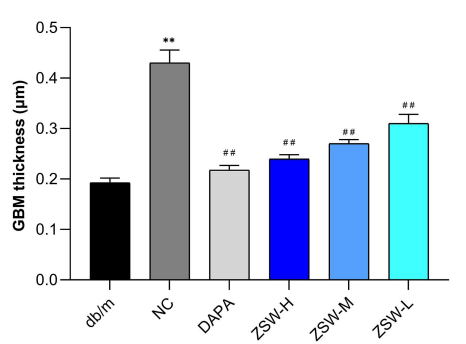

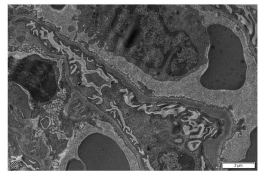

ZSW-H
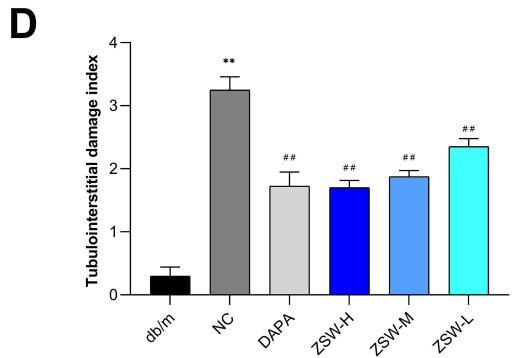

Figure 7 ZSW treatment alleviates renal pathological abnormalities in db/db mice. (A) Observation by light microscopy (HE, PAS, and Masson staining, $400 \times$ ) and TEM $(6000 \times)$ in renal tissues of $\mathrm{db} / \mathrm{db}$ mice. GBM thickening (shown in Masson staining and TEM), mesangial matrix expansion (shown in PAS staining and TEM) and tubular inflammation (shown in HE staining) are indicated by yellow arrows. ZSW treatment decreases percentage of glomerular collagenous fiber (B), GBM thickness (C), and

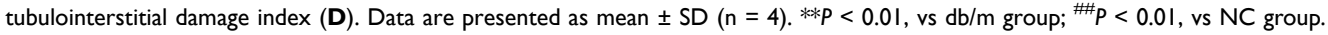

differentiation, proliferation, apoptosis and migration of cells. However, this pathway can be excessively activated by hyperglycemia, which results in cell dysfunction, accelerates ECM accumulation and EMT in renal tubular epithelial cells, eventually promotes glomerulosclerosis and renal interstitial fibrosis. ${ }^{50}$ We found that both the MAPK and PI3K-Akt signaling pathways were among the top $50 \mathrm{KEGG}$ pathways; moreover, the genes encoding the related targets were the key targets of $\mathrm{ZSW}$ in DN treatment. In addition, our animal study indicated that ZSW can decrease the phosphoprotein and mRNA levels of p38 and Akt in $\mathrm{db} / \mathrm{db}$ mice, revealing that ZSW exerted its therapeutic effects on DN partially by regulating the related signaling pathways.

It is worth noting that $\mathrm{ZSW}$ exerted renoprotective effects via targets and pathways related to many other molecular mechanisms besides inflammation as shown by network pharmacology analysis. Vascular endothelial growth factor (VEGF) signaling pathway has been confirmed to play a central role in the pathogenesis of microvascular complications of diabetes including $\mathrm{DN}$, as causing diabetic hyperfiltration and albuminuria. ${ }^{51-53}$ The Ras proteins are GTPases that function as molecular switches for signaling pathways regulating cell proliferation, survival, growth, migration, differentiation or cytoskeletal dynamism. A previous study has shown that renal mesangial cells increase Ras activation and mediate intracellular signal transduction to promote VEGF gene transcription when stimulated by high glucose. ${ }^{54}$ AGEs are a complex group of compounds produced through the nonenzymatic glycation and oxidation of proteins, lipids and nucleic acids. Receptor for AGE (RAGE) mediated by AGEs can activate diverse signal transduction cascades and downstream pathways, including generation of 


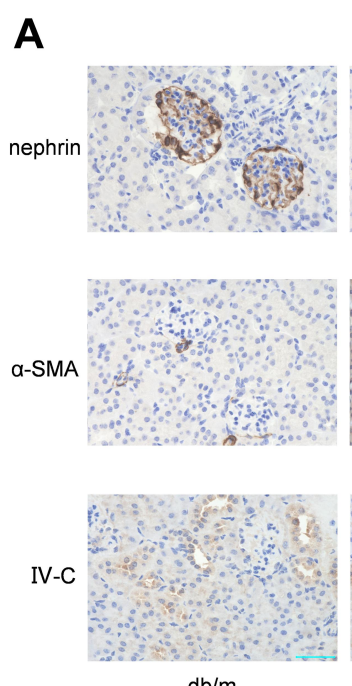

$\mathrm{db} / \mathrm{m}$
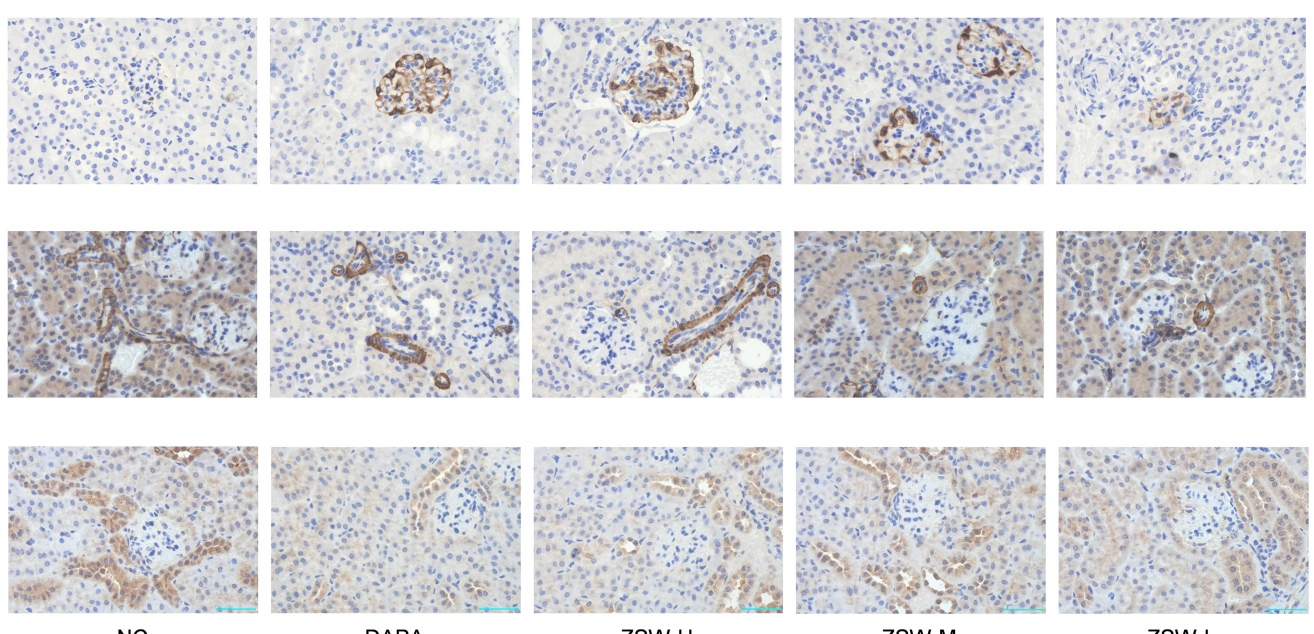

NC

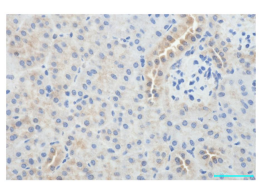

DAPA

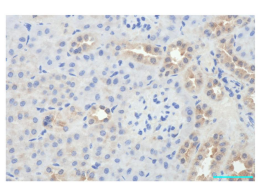

ZSW-H

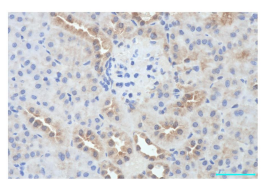

ZSW-M

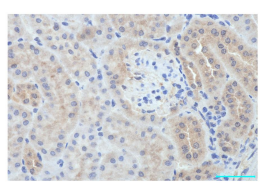

ZSW-L
B1

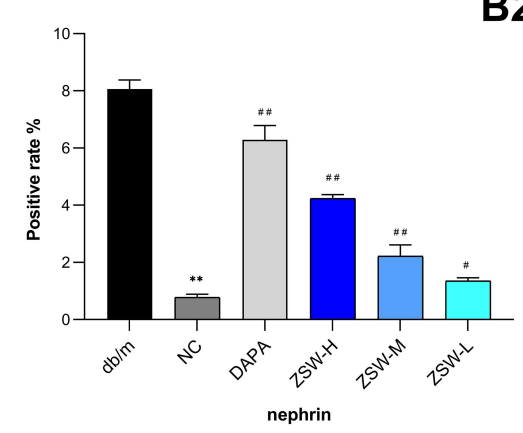

B2

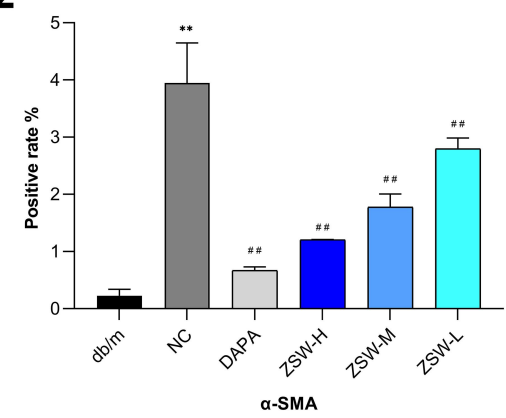

B3

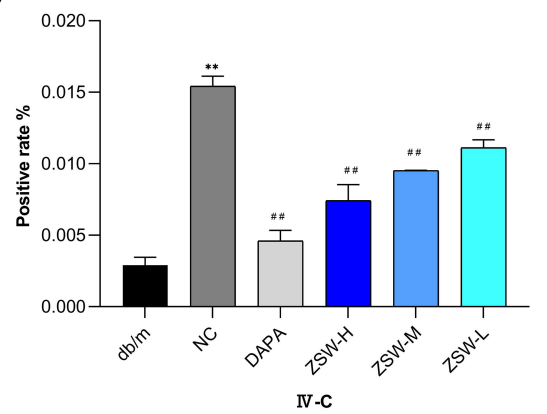

Figure $8 \mathrm{ZSW}$ treatment regulates the major biomarkers of renal injuries in db/db mice. (A) Nephrin, $\alpha$-SMA and IV-C expression detected by IHC analysis (400 $\times$ ); (B) The positive rate of the IHC results. Data are presented as mean $\pm \mathrm{SD}(\mathrm{n}=3) . * * P<0.0 \mathrm{I}$, vs $\mathrm{db} / \mathrm{m}$ group; ${ }^{\#} P<0.05$, ${ }^{\# *} P<0.0 \mathrm{I}$, vs $\mathrm{NC}$ group.

reactive oxygen species (ROS). ${ }^{55}$ Interaction of AGEs with RAGE stimulates could contribute to progressive alteration in renal architecture such as mesangial matrix expansion and GBM thickening and sclerosis, which lead to renal dysfunction. ${ }^{56-58}$ The role of ZSW in these crucial mechanisms of DN have been demonstrated by network pharmacology analysis in the current study, which requires further experimental verifications and explorations.

\section{Conclusion}

In conclusion, according to network pharmacology analysis, the effective mechanisms of ZSW in DN therapy involve the regulation of targets and pathways in multiple biological processes, particularly inflammation. The antiinflammatory activity of ZSW in $\mathrm{db} / \mathrm{db}$ mice seems to be mediated by inhibition of the exaggerated release of inflammatory cytokines including IL- $1 \beta$, IL-6, TNF- $\alpha$, and MCP-1 as well as regulation of p38 MAPK and PI3K-Akt signaling pathways. ZSW might be used as a potent therapeutic agent for DN.

\section{Abbreviations}

DN, diabetic nephropathy; ESRD, end-stage renal disease; ZSW, ZiShenWan; PPI, protein-protein interaction; GO, gene ontology; KEGG, Kyoto encyclopedia of genes and genomes; IL, interleukin; TNF, tumor necrosis factor receptor; MCP, monocyte chemotactic protein; MAPK, mitogen activated protein kinases; PI3K, phosphoinositide 3-kinase; Akt, protein kinase B; GBM, glomerular basement membrane; ECM, extracellular matrix; EMT, epithelial-mesenchymal transition; JAK-STAT, Janus kinase/ signal transducers and activators of transcription; NF- $\mathrm{KB}$, nuclear transcription factor kappa B; Nrf2, nuclear factor erythroid 2-related factor 2; TCM, traditional Chinese medicine; TCMSP, Traditional Chinese Medicine Systems Pharmacology; ADME, absorption, distribution, metabolism, and excretion; OB, oral bioavailability; DL, drug similarity; CTD, Comparative Toxicogenomic Database; TTD, Therapeutic Target Database; OMIM, Online Mendelian Inheritance in Man; GAD, Genetic 
A

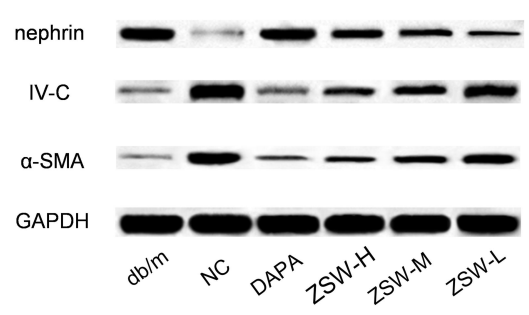

B1

C1
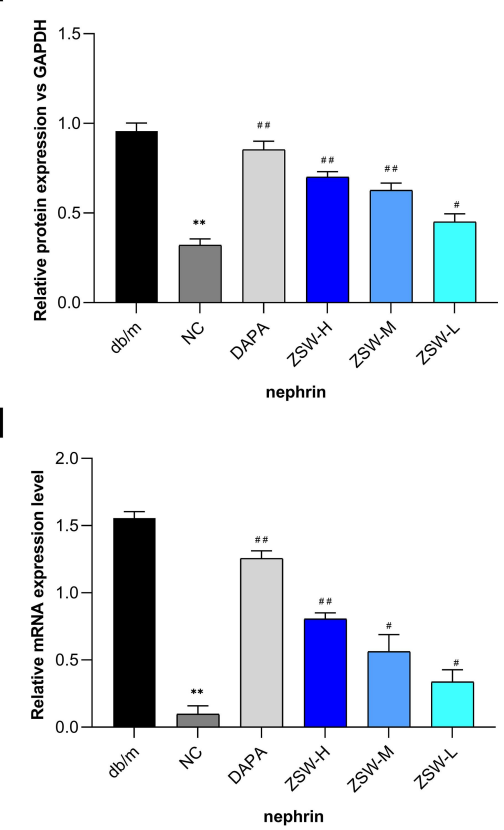

B2

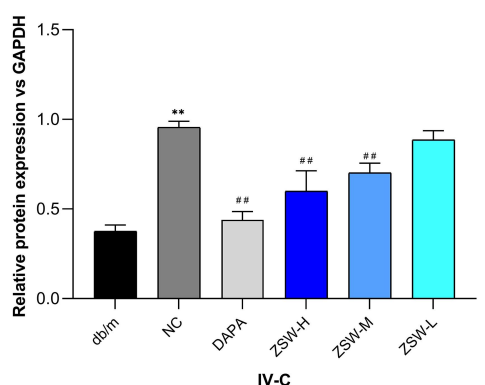

C2

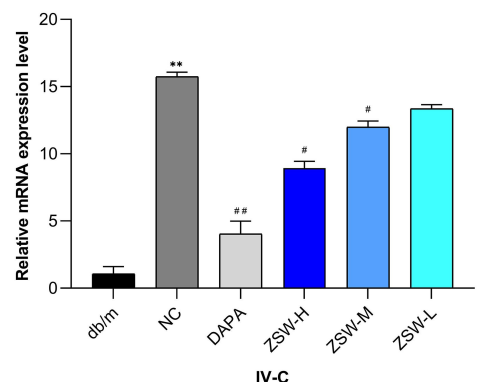

B3

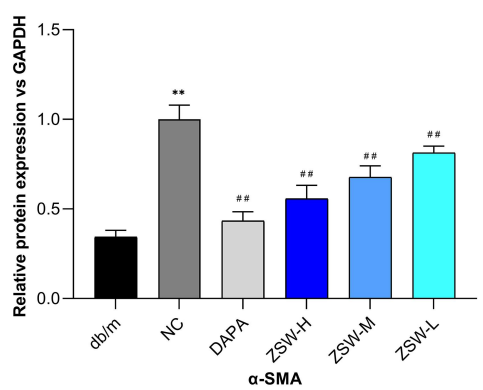

C3

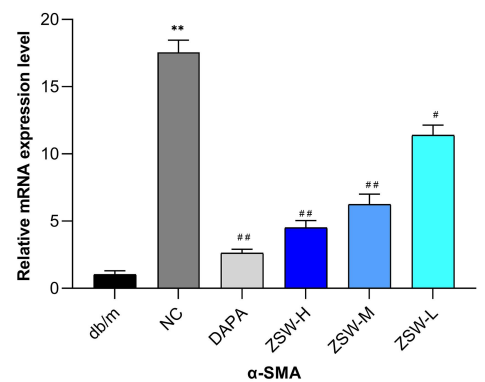

Figure $9 \mathrm{ZSW}$ treatment regulates protein and mRNA expressions of nephrin, IV-C and $\alpha$-SMA in db/db mice. Representative band of nephrin, IV-C and $\alpha$-SMA protein detected by WB (A). The relative protein levels of nephrin (BI), IV-C (B2) and $\alpha$-SMA (B3) of the WB results; The levels of nephrin (CI), IV-C (C2) and $\alpha-S M A(C 3)$ mRNA assessed by RT-qPCR. Data are presented as mean \pm SD $(n=3)$. ${ }^{* * p}<0.01$, vs $\mathrm{db} / \mathrm{m}$ group; ${ }^{\#} P<0.05,{ }^{\# \#} P<0.01$, vs $N C$ group.

A

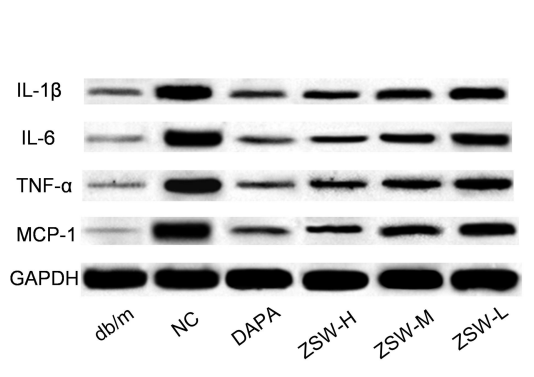

B

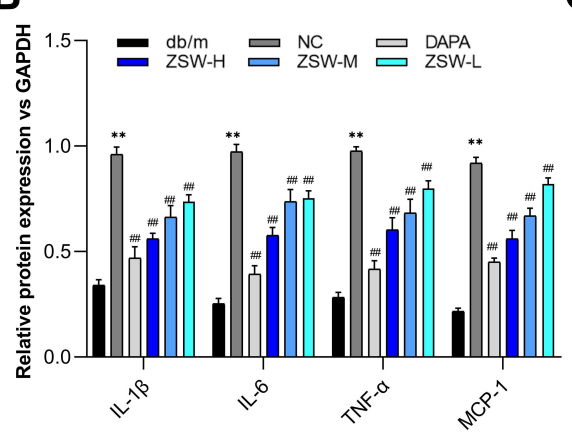

C

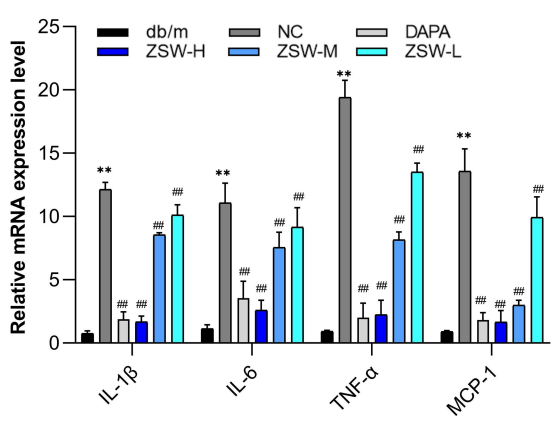

Figure 10 ZSW treatment inhibits expression of multiple proinflammatory cytokines exaggerated in db/db mice. (A) Representative band of IL-I $\beta$, IL-6, TNF- $\alpha$, and MCP-I protein detected by WB. (B) The relative protein levels of the WB results; (C) The levels of IL-I $\beta$, IL-6, TNF- $\alpha$, and MCP-I mRNA assessed by RT-qPCR. Data are presented as mean $\pm \mathrm{SD}(\mathrm{n}=3)$. ${ }^{* * P}<0.0 \mathrm{I}$, vs $\mathrm{db} / \mathrm{m}$ group; ${ }^{\# \#} \mathrm{P}<0.0 \mathrm{I}$, vs $\mathrm{NC}$ group.

Association Database; PHARMGKB, Pharmacogenomics

Knowledge Base; BP, biological process; CC, cellular component; MF, molecular function; DAVID, Database for Annotation, Visualization and Integrated Discovery;

DAPA, dapagliflozin; SGLT2, sodium-glucose cotransporter 2 ; $\alpha$-SMA, $\alpha$-smooth muscle actin; GAPDH, 


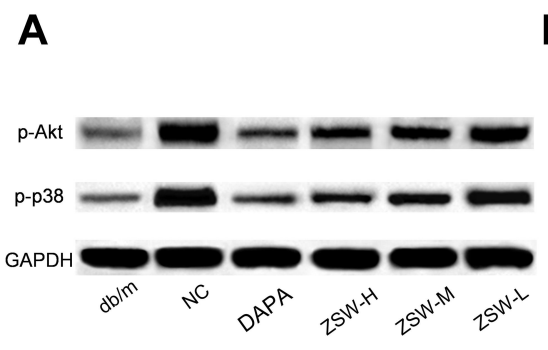

B

C
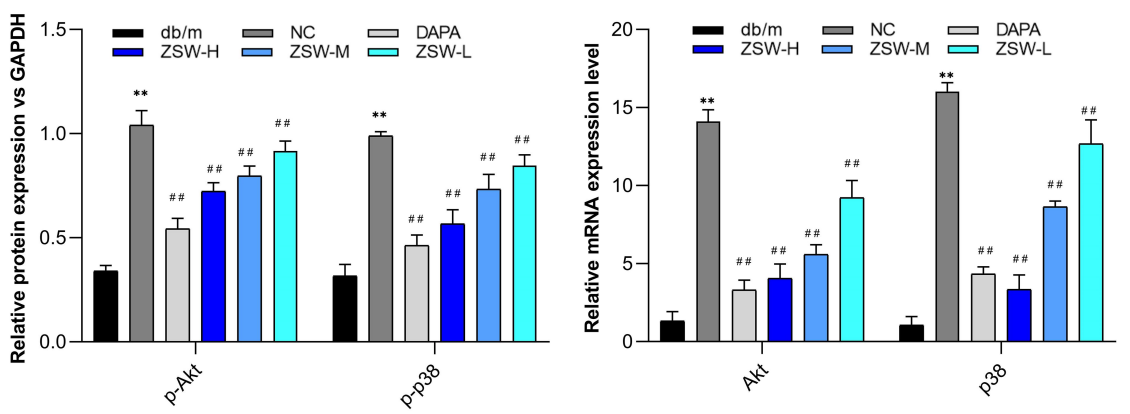

Figure II ZSW treatment regulates the expression of key nodes in PI3K-Akt and p38 MAPK signaling pathways activated in db/db mice. (A) Representative band of p-Akt and p-p38 protein detected by WB. (B) The relative protein levels of the WB results; (C) The levels of Akt and p38 MAPK mRNA assessed by RT-qPCR. Data are presented as mean $\pm \mathrm{SD}(\mathrm{n}=3)$. ${ }^{* * P} P<0.0 \mathrm{I}$, vs $\mathrm{db} / \mathrm{m}$ group; ${ }^{\# \#} \mathrm{P}<0.0 \mathrm{I}$, vs $\mathrm{NC}$ group.

glyceraldehyde-3-phosphate dehydrogenase; IV-C, type IV collagen; NGAL, neutrophil gelatinase-associated lipocalin; ELISA, enzyme-linked immunosorbent assay; $\mathrm{NC}$, non-treated control; ZSW-H, high-dose ZiShenWan; ZSW-M, medium-dose ZiShenWan; ZSW-L, lowdose ZiShenWan; FBG, fasting blood glucose; WB, Western blotting; RT-qPCR, real-time quantitative polymerase chain reaction; CRE, creatinine; BUN, blood urea nitrogen; ACR, albumin concentration versus creatinine concentration ratio; HE, hematoxylin and eosin; PAS, periodic acid-Schiff; TEM, transmission electron microscopy; IHC, immunohistochemistry; SD, standard deviation; ANOVA, analysis of variance; LSD, least significant difference; AGEs, advanced glycosylation end products; VEGF, vascular endothelial growth factor; RAGE, Receptor for AGE; ROS, reactive oxygen species.

\section{Acknowledgments}

This work was supported by the International Cooperation Project of State Administration of Traditional Chinese Medicine (No. GZYYGJ2019034).

\section{Disclosure}

The authors declare that there are no conflicts of interest in this work.

\section{References}

1. Zhang L, Long J, Jiang W, et al. Trends in chronic kidney disease in China. $N$ Engl $J$ Med. 2016;375(9):905-906. doi:10.1056/ NEJMc1602469

2. Wen CP, Chang CH, Tsai MK, et al. Diabetes with early kidney involvement may shorten life expectancy by 16 years. Kidney Int. 2017;92(2):388-396. doi:10.1016/j.kint.2017.01.030
3. Lewis EJ, Lewis JB. Treatment of diabetic nephropathy with angiotensin II receptor antagonist. Clin Exp Nephrol. 2003;7(1):1-8. doi: $10.1007 / \mathrm{s} 101570300000$

4. Gallagher H, Suckling RJ. Diabetic nephropathy: where are we on the journey from pathophysiology to treatment? Diabetes Obes Metab. 2016;18(7):641-647. doi:10.1111/dom.12630

5. Donate-Correa J, Luis-Rodríguez D, Martín-Núñez E, et al. Inflammatory targets in diabetic nephropathy. J Clin Med. 2020;9 (2):458. doi:10.3390/jcm9020458

6. Li G. [LanShiMiCang]. In: Wen Q, Ding GH, eds. Beijing: People's Medical Publishing House; 2012.

7. Liang G, Tang H, Ni D, et al. Zishenwan decreases kidney damage in recurrent urinary tract infection through the inhibition of toll-like receptor 4 signal. Evid Based Complement Alternat Med. 2018;2018:5968657. doi:10.1155/2018/5968657

8. Li Z, Qi Z. [Analysis on the pathogenesis of diabetes from Shi Jinmo's medicine couples]. Beijing J Tradit Chin Med. 2012;31 (1):28-29, 72. Chinese. doi:10.16025/j.1674-1307.2012.01.009

9. Miura T, Ichiki H, Iwamoto $\mathrm{N}$, et al. Antidiabetic activity of the rhizoma of Anemarrhena asphodeloides and active components, mangiferin and its glucoside. Biol Pharm Bull. 2001;24(9):1009-1011. doi:10.1248/bpb.24.1009

10. Kim HJ, Kong MK, Kim YC. Beneficial effects of Phellodendri Cortex extract on hyperglycemia and diabetic nephropathy in streptozotocin-induced diabetic rats. BMB Rep. 2008;41 (10):710-715. doi:10.5483/bmbrep.2008.41.10.710

11. Yan YM, Fang P, Yang MT, Li N, Lu Q, Cheng YX. Anti-diabetic nephropathy compounds from Cinnamomum cassia. J Ethnopharmacol. 2015;165:141-147. doi:10.1016/j.jep.2015.01.049

12. Tang YH, Sun ZL, Fan MS, Li ZX, Huang CG. Anti-diabetic effects of TongGuanWan, a Chinese traditional herbal formula, in C57BL/KsJ-db/ db mice. Planta Med. 2012;78(1):18-23. doi:10.1055/s-0031-1280268

13. Zhu X, Cheng YQ, Du L, et al. Mangiferin attenuates renal fibrosis through down-regulation of osteopontin in diabetic rats. Phytother Res. 2015;29:295-302. doi:10.1002/ptr.5254

14. Ma X, Chen Z, Wang L, et al. The pathogenesis of diabetes mellitus by oxidative stress and inflammation: its inhibition by berberine. Front Pharmacol. 2018;9:782. doi:10.3389/fphar.2018.00782

15. Liao JC, Deng JS, Chiu CS, et al. Anti-inflammatory activities of cinnamomum cassia constituents in vitro and in vivo. Evid Based Complement Alternat Med. 2012;2012:429320. doi:10.1155/2012/429320

16. Zhang GB, Li QY, Chen QL, Su SB. Network pharmacology: a new approach for chinese herbal medicine research. Evid Based Complement Alternat Med. 2013;2013:621423. doi:10.1155/2013/621423

17. Ru J, Li P, Wang J, et al. TCMSP: a database of systems pharmacology for drug discovery from herbal medicines. $J$ Cheminform. 2014;6:13. doi:10.1186/1758-2946-6-13 
18. Davis AP, Grondin CJ, Johnson RJ, et al. The comparative toxicogenomics database: update 2019. Nucleic Acids Res. 2019;47 (D1):948-954. doi:10.1093/nar/gky868

19. Wang Y, Zhang S, Li F, et al. Therapeutic target database 2020: enriched resource for facilitating research and early development of targeted therapeutics. Nucleic Acids Res. 2020;48(D1):1031-1041. doi:10.1093/nar/gkz981

20. Wishart DS, Feunang YD, Guo AC, et al. DrugBank 5.0: a major update to the DrugBank database for 2018. Nucleic Acids Res. 2018;46(D1):1074-1082. doi:10.1093/nar/gkx1037

21. Hamosh A, Scott AF, Amberger JS, Bocchini CA, McKusick VA. Online mendelian inheritance in man (OMIM), a knowledgebase of human genes and genetic disorders. Nucleic Acids Res. 2005;33 (D):514-517. doi:10.1093/nar/gki033

22. Becker KG, Barnes KC, Bright TJ, Wang SA. The genetic association database. Nat Genet. 2004;36(5):431-432. doi:10.1038/ng0504-431

23. Whirl-Carrillo M, McDonagh EM, Hebert JM, et al. Pharmacogenomics knowledge for personalized medicine. Clin Pharmacol Ther. 2012;92(4):414-417. doi:10.1038/clpt.2012.96

24. Morgat A, Lombardot T, Coudert E, et al. Enzyme annotation in UniProtKB using Rhea. Bioinformatics. 2020;36(6):1896-1901. doi:10.1093/bioinformatics/btz817

25. Otasek D, Morris JH, Bouças J, Pico AR, Demchak B. Cytoscape automation: empowering workflow-based network analysis. Genome Biol. 2019;20(1):185. doi:10.1186/s13059-019-1758-4

26. Szklarczyk D, Gable AL, Lyon D, et al. STRING v11: protein-protein association networks with increased coverage, supporting functional discovery in genome-wide experimental datasets. Nucleic Acids Res. 2019;47(D1):607-613. doi:10.1093/nar/gky1131

27. Huang da W, Sherman BT, Lempicki RA. Systematic and integrative analysis of large gene lists using DAVID bioinformatics resources. Nat Protoc. 2009;4(1):44-57. doi:10.1038/nprot.2008.211

28. Jaikumkao K, Pongchaidecha A, Chueakula N, et al. Dapagliflozin, a sodium-glucose co-transporter-2 inhibitor, slows the progression of renal complications through the suppression of renal inflammation, endoplasmic reticulum stress and apoptosis in prediabetic rats. Diabetes Obes Metab. 2018;20(11):2617-2626. doi:10.1111/dom.13441

29. ElMahdy MK, Helal MG, Ebrahim TM. Potential anti-inflammatory effect of dapagliflozin in HCHF diet-induced fatty liver degeneration through inhibition of $\mathrm{TNF}-\alpha$, IL-1 $\beta$, and IL-18 in rat liver. Int Immunopharmacol. 2020;86:106730. doi:10.1016/j. intimp.2020.106730

30. Liu H, Zhao L, Zhang J, et al. Critical role of cysteine-rich protein 61 in mediating the activation of renal fibroblasts. Front Physiol. 2019;10:464. doi:10.3389/fphys.2019.00464

31. Yiu WH, Wong DW, Wu HJ, et al. Kallistatin protects against diabetic nephropathy in $\mathrm{db} / \mathrm{db}$ mice by suppressing AGE-RAGE induced oxidative stress. Kidney Int. 2016;89(2):386-398. doi:10.1038/ki.2015.331

32. Kanwar YS, Sun L, Xie P, Liu FY, Chen S. A glimpse of various pathogenetic mechanisms of diabetic nephropathy. Annu Rev Pathol. 2011;6:395-423. doi:10.1146/annurev.pathol.4.110807.092150

33. Ward MG, Li G, Barbosa-Lorenzi VC, Hao M. Stigmasterol prevents glucolipotoxicity induced defects in glucose-stimulated insulin secretion. Sci Rep. 2017;7(1):9536. doi:10.1038/s41598-017-10209-0

34. Lin CF, Kuo KT, Chen TY, Chien CT. Quercetin-rich Guava (Psidium Guajava) juice in combination with Trehalose reduces autophagy, apoptosis and pyroptosis formation in the kidney and pancreas of type II diabetic rats. Molecules. 2016;21(3):334. doi:10.3390/ molecules21030334

35. Liu WH, Hei ZQ, Nie H, et al. Berberine ameliorates renal injury in streptozotocin-induced diabetic rats by suppression of both oxidative stress and aldose reductase. Chin Med $J$ (Engl). 2008;121 (8):706-712. doi:10.1097/00029330-200804020-00009
36. Zhang J, Zhang R, Wang Y, et al. Effects of neutrophil-lymphocyte ratio on renal function and histologic lesions in patients with diabetic nephropathy. Nephrology (Carlton). 2019;24(11):1115-1121. doi:10.1111/nep.13517

37. Zou LX, Sun L. Global diabetic kidney disease research from 2000 to 2017: a bibliometric analysis. Medicine (Baltimore). 2019;98(6): e14394. doi:10.1097/MD.0000000000014394

38. Chen H, Charlat O, Tartaglia LA, et al. Evidence that the diabetes gene encodes the leptin receptor: identification of a mutation in the leptin receptor gene in $\mathrm{db} / \mathrm{db}$ mice. Cell. 1996;84(3):491-495. doi:10.1016/s0092-8674(00)81294-5

39. Sharma K, McCue P, Dunn SR. Diabetic kidney disease in the $\mathrm{db} / \mathrm{db}$ mouse. Am J Physiol Renal Physiol. 2003;284(6):F1138- F1144. doi:10.1152/ajprenal.00315.2002

40. Nowak N, Skupien J, Smiles AM, et al. Markers of early progressive renal decline in type 2 diabetes suggest different implications for etiological studies and prognostic tests development. Kidney Int. 2018;93(5):1198-1206. doi:10.1016/j.kint.2017.11.024

41. Tang SC, Lai KN. The pathogenic role of the renal proximal tubular cell in diabetic nephropathy. Nephrol Dial Transplant. 2012;27 (8):3049-3056. doi:10.1093/ndt/gfs260

42. Tung CW, Hsu YC, Shih YH, Chang PJ, Lin CL. Glomerular mesangial cell and podocyte injuries in diabetic nephropathy. Nephrology (Carlton). 2018;23(Suppl 4):32-37. doi:10.1111/nep.13451

43. Okonogi H, Nishimura M, Utsunomiya Y, et al. Urinary type IV collagen excretion reflects renal morphological alterations and type IV collagen expression in patients with type 2 diabetes mellitus. Clin Nephrol. 2001;55(5):357-364.

44. Essawy M, Soylemezoglu O, Muchaneta-Kubara EC, Shortland J, Brown CB, El Nahas AM. Myofibroblasts and the progression of diabetic nephropathy. Nephrol Dial Transplant. 1997;12(1):43-50. doi:10.1093/ndt/12.1.43

45. Li J, Qu X, Bertram JF. Endothelial-myofibroblast transition contributes to the early development of diabetic renal interstitial fibrosis in streptozotocin-induced diabetic mice. Am J Pathol. 2009;175 (4):1380-1388. doi:10.2353/ajpath.2009.090096

46. Chen YL, Qiao YC, Xu Y, et al. Serum TNF- $\alpha$ concentrations in type 2 diabetes mellitus patients and diabetic nephropathy patients: a systematic review and meta-analysis. Immunol Lett. 2017;186:52-58. doi:10.1016/j.imlet.2017.04.003

47. Park J, Ryu DR, Li JJ, et al. MCP-1/CCR2 system is involved in high glucose-induced fibronectin and type IV collagen expression in cultured mesangial cells. Am J Physiol Renal Physiol. 2008;295(3): F749-F757. doi:10.1152/ajprenal.00547.2007

48. Cargnello M, Roux PP. Activation and function of the MAPKs and their substrates, the MAPK-activated protein kinases. Microbiol Mol Biol Rev. 2011;75(1):50-83. doi:10.1128/MMBR.00031-10

49. Ma FY, Liu J, Nikolic-Paterson DJ. The role of stress-activated protein kinase signaling in renal pathophysiology. Braz J Med Biol Res. 2009;42(1):29-37. doi:10.1590/s0100-879x2008005000049

50. Rane MJ, Song Y, Jin S, et al. Interplay between Akt and p38 MAPK pathways in the regulation of renal tubular cell apoptosis associated with diabetic nephropathy. Am J Physiol Renal Physiol. 2010;298(1): F49-F61. doi:10.1152/ajprenal.00032.2009

51. Flyvbjerg A. Putative pathophysiological role of growth factors and cytokines in experimental diabetic kidney disease. Diabetologia. 2000;43(10):1205-1223. doi:10.1007/s001250051515

52. Tilton RG, Kawamura T, Chang KC, et al. Vascular dysfunction induced by elevated glucose levels in rats is mediated by vascular endothelial growth factor. J Clin Invest. 1997;99(9):2192-2202. doi:10.1172/JCI119392

53. Sung SH, Ziyadeh FN, Wang A, Pyagay PE, Kanwar YS, Chen S. Blockade of vascular endothelial growth factor signaling ameliorates diabetic albuminuria in mice. $J$ Am Soc Nephrol. 2006;17 (11):3093-3104. doi:10.1681/ASN.2006010064 
54. Ho C, Hsu YC, Tseng CC, et al. Simvastatin alleviates diabetes-induced VEGF-mediated nephropathy via the modulation of Ras signaling pathway. Ren Fail. 2008;30(5):557-565. doi: $10.1080 / 08860220802064457$

55. Tan AL, Forbes JM, Cooper ME. AGE, RAGE, and ROS in diabetic nephropathy. Semin Nephrol. 2007;27(2):130-143. doi:10.1016/j. semnephrol.2007.01.006

56. Yamamoto Y, Kato I, Doi T, et al. Development and prevention of advanced diabetic nephropathy in RAGE-overexpressing mice. J Clin Invest. 2001;108(2):261-268. doi:10.1172/JCI11771
57. Reiniger N, Lau K, McCalla D, et al. Deletion of the receptor for advanced glycation end products reduces glomerulosclerosis and preserves renal function in the diabetic OVE26 mouse. Diabetes. 2010;59(8):2043-2054. doi:10.2337/db09-1766

58. Wendt TM, Tanji N, Guo J, et al. RAGE drives the development of glomerulosclerosis and implicates podocyte activation in the pathogenesis of diabetic nephropathy. Am J Pathol. 2003;162 (4):1123-1137. doi:10.1016/S0002-9440(10)63909-0

\section{Publish your work in this journal}

Drug Design, Development and Therapy is an international, peerreviewed open-access journal that spans the spectrum of drug design and development through to clinical applications. Clinical outcomes, patient safety, and programs for the development and effective, safe, and sustained use of medicines are a feature of the journal, which has also been accepted for indexing on PubMed Central. The manuscript management system is completely online and includes a very quick and fair peer-review system, which is all easy to use. Visit http://www. dovepress.com/testimonials.php to read real quotes from published authors. 